\title{
Blood Vessels Detection and Segmentation in Retina using Gabor Filters
}

\section{Farnaz Farokhian}

Submitted to the

Institute of Graduate Studies and Research

in partial fulfillment of the requirements for the Degree of

\author{
Master of Science \\ in \\ Electrical and Electronic Engineering
}

Eastern Mediterranean University

September 2013

Gazimağusa, North Cyprus 
Approval of the Institute of Graduate Studies and Research

Prof. Dr. Elvan Yilmaz

Director

I certify that this thesis satisfies the requirements as a thesis for the degree of Master of Science in Electrical and Electronic Engineering.

Prof. Dr. Aykut Hocanın

Chair, Department of Electrical and Electronic Engineering

We certify that we have read this thesis and that in our opinion, it is fully adequate, in scope and quality, as a thesis of the degree of Master of Science in Electrical and Electronic Engineering.

Assoc. Prof. Dr. Hasan Demirel

Supervisor

Examining Committee

1. Prof. Dr. Hüseyin Özkaramanlı

2. Prof. Dr. Sener Uysal

3. Assoc. Prof. Dr. Hasan Demirel 


\begin{abstract}
Currently, medical images have become an important field of research due to the progress in their acquisition, storage and management in a wide range of applications. In the medical domain, a huge effort has been devoted to develop applications and tools related with disease diagnosis and identification of anatomical structures. Spatially, the extraction of the vessel pattern from retinal images has an important issue not only in the medical tools; but also in biometric identification applications. A lot of research has been devoted to accurately extract the retinal vessels in order to define applications for early ocular disease diagnosis. The main regions of retina are optic disk, fovea and blood vessels. The identification of these areas can help in analyses of the diseases that affect these regions such as retinopathy of prematurity, glaucoma and diabetic retinopathy.
\end{abstract}

In earlier studies the Gabor filters have received considerable attention because of the characteristics of certain cells in the visual cortex of some mammals can be defined by these filters. In addition, Gabor has shown that the representation of signals using the Gaussian functions modulated by complex exponentials is optimal in the sense of minimizing the joint uncertainty in the combined time-frequency domain. Therefore, these functions provide the best trade-off between time resolution and frequency resolution.

In this thesis we propose to use Gabor filtering technique for the detection of blood vessels in retina. For retina images we used 180 different filters, with the rotation separation of 1 degree to capture for more edge information of the vessels. Different 
scales are also used to include edge information of the vessels. After Gabor filtering image processing methods including logarithmic normalization, morphological erosion, mask processing and thresholding are used to perform the segmentation of the detected vessels. The proposed approach is applied to retina images from publicly available databases which is the Digital Retinal Images for Vessel Extraction database (DRIVE). Manually segmented versions of these images along with masks are used for performance analysis.

Receiver Operating Characteristic (ROC) Curves containing false acceptance rate (FAR) and false rejection rate (FRR) are incorporated to systematically determine a suitable threshold for reliable segmentation performance. Sensitivity, specificity, efficiency and accuracy of the retina vessels segmentation have been studied and the generated results are comparable with alternative state-of-the-art methods available in the relevant literature. The results show that the employed method produces comparable and sometimes better results than the alternative methods available in the literature.

Keywords: Digital Image Processing, Gabor Filters, Detection of Blood Vessels, Edge Detection, Morphological Filters, Retinal Fundus Images 


\section{ÖZ}

Günümüzde, medikal imge araştırmaları, veri edinim, depolama ve kullanma gibi alanlarda kaydedilen gelişmelerden dolayı önemli bir araştırma alanı haline gelmiştir. Tıbbi alanda, anatomik yapıların hastalığın tanımı ve teşhisi ile ilgili uygulamalar ve araçların geliştirilebilmesi için büyük çabalar ortaya konulmuştur. Göz retinasının şekil olarak yapısın incelenmesi ve tanımlanması sadece tıp alanında değil biyometrik kimlik tanımlama uygulamalarında da önemli aşamalat katedilmiştir. Retinadan hastaliğın tam olarak erken teşhis ve tespitini sağlayacak pek çok bilimsel araştırmalar yapılmıştır. Retinadaki ana kısımlar optik yuvarlak, gözbebegi ve retinadaki damarlardir. Bu kısımların incelenmesi, prematüre retinopati, glokom ve diyabetik retinopati gibi bu bölgeleri etkileyen hastalıkların analizleri ve teşhisi için yardımcı olmaktadır.

İlk yapılan çalışmalarda Gabor filtreleri, bazı memelilerin görsel korteksindeki hücrelerin özelliklerinin tanınmasında başarılı olduklarından dolayı, oldukça fazla dikkat çekmişlerdi. Buna ek olarak, Gabor karmaşık üstel tarafından modüle edilmiş olan Gauss fonksiyonu tabanlı sinyallerin zaman-frekans etki alanında ortak belirsizliklerinin en aza indirerek optimal bir şekilde temsil edebildiğini göstermiştir. $\mathrm{Bu}$ nedenle bu fonksiyonlar zaman cözurlüğ̈ ile frekans çözünürlüğünün en iyi şekilde dengelenmesini sağlamaktadır.

$\mathrm{Bu}$ tezde retinadaki kan damarlarının tespiti için Gabor filtreleme tekniği kullanmayı öneriyoruz. Retina imgelerine ait etkin bilgileri sağlayabilmek için yüksek frekanslı kenar kısımlardan bu bilgileri edinelbilmek doğrultusunda dönel 1 derece ayrimli 
180 değişik filitre kullandık. Farklı ölçekleri de damarların kenar bilgilerini dahil etmek kullanılmıştır. Gabor filitre yöntemi uygulamasından sonra logaritmik düzgeleme, morfolojik erozyon, maskeleme ve eşikleme asamaları damarların bölütlenmesinin gerçekleştirilmesi için kullanılmıştır. Önerilen yöntem, kamuya açık retina bilgileri ile oluşturulmuş sayısal retina görüntü damar çıkarımı (DRIVE) veri tabanına bulunan retina imgelerine uygulanmıştır. Kullanılan retina imgelerine ait manuel olarak bölütlenmiş versiyonları da performans analizi süreçlerinde kullanılmıştır.

Yanlış kabul oranı (FAR) ve yanlış red oranı (FRR) içeren alıcı çalışma karakteristiği (ROC) eğrileri sistematik güvenilir bölümleme performansı çalışmasına yönelik olarak uygun bir eşik belirlemek için dahil edilmiştir. Duyarlılık, özgüllük, verimlilik ve retina damarlarının bölümleme doğruluğu çalışılmış ve elde edilen sonuçlar ilgili literatürde mevcut alternatif yöntemlerle karşılaştırılmıştır. Sonuçlar, kullanılan yöntemin, literatürde mevcut olan alternatif yöntemler ile karşılaştırılabilir ve bazen de daha iyi sonuçlar verdiğini göstermektedir.

Anahtar Kelimeler: Dijital görüntü işleme, Gabor filtreleri, kan damarlarının sezimi, kenar sezimi, morfolojik filtreler, retinal fundus imgeleri. 
Dedicated to

My husband who has always been supportive of me during my time at EMU and

my parents. 


\section{ACKNOWLEDGMENTS}

First and foremost I would like to thank my supervisor Assoc. Prof. Dr. Hasan Demirel for guiding and helping me in my master study, for his patience and sharing kindly his knowledge with me.

I also wish to thank all the faculty members at the department of Electrical and Electronic Engineering, and specially the chairman, Prof. Dr. Aykut Hocanın, for providing a conductive environment during my master studies.

Last but not least, I would like to express my appreciation to my husband and parents who have always given me support and encouragement. 


\section{TABLE OF CONTENTS}

ABSTRACT

ÖZ V

ACKNOWLEDGMENTS VIII

TABLE OF CONTENTS IX

LIST OF FIGURES XII

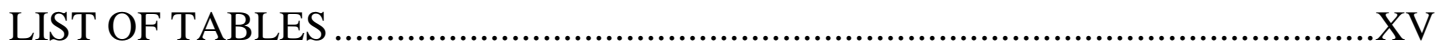

LIST OF SYMBOLS AND ABBREVIATIONS ............................................ XVI

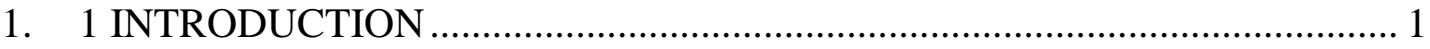

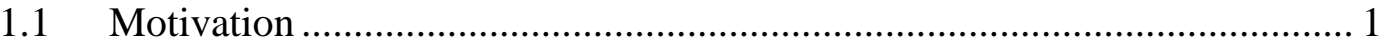

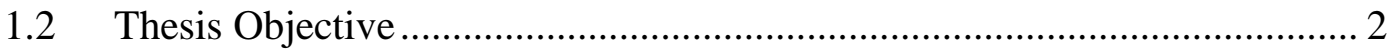

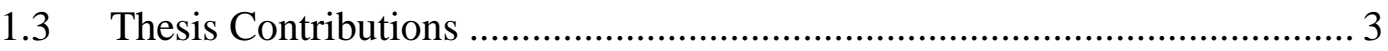

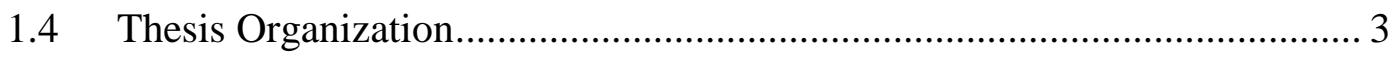

2. USING RETINAL BLOOD VESSELS ANAYSIS FOR DISEASE

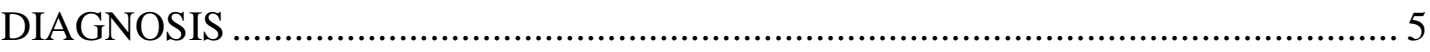

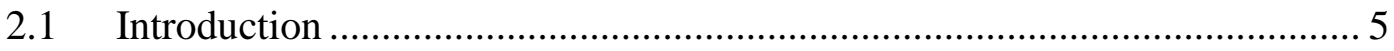

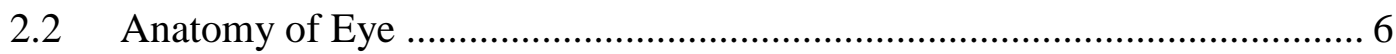

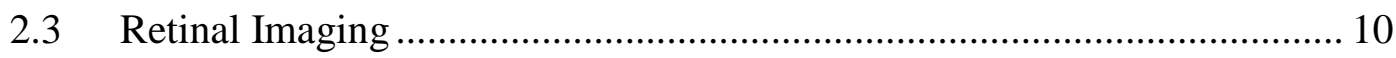

2.4 Vessel Properties and their Applications ............................................. 11

3. GABOR FILTERS AND THEIR APPLICATIONS …................................... 13

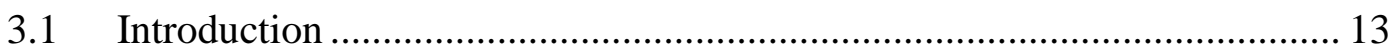




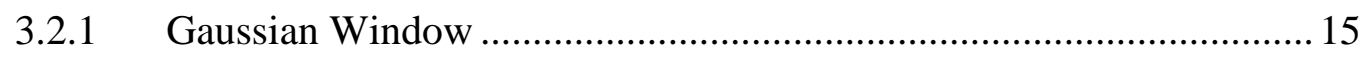

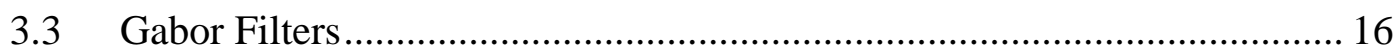

3.3.1 One-dimensional Gabor Filter ..................................................... 17

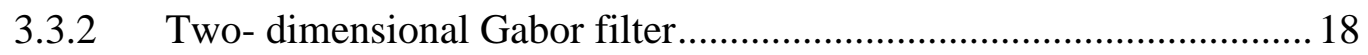

3.4 The Complex Gabor Function in Space Domain: ................................... 20

3.4.1 Half-magnitude Profile .............................................................. 23

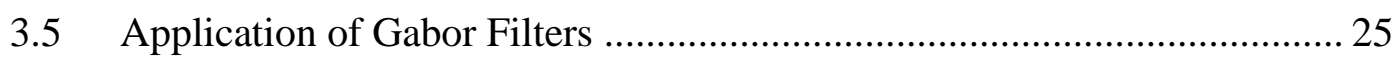

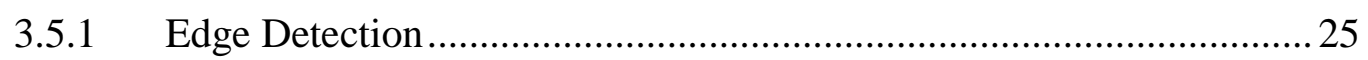

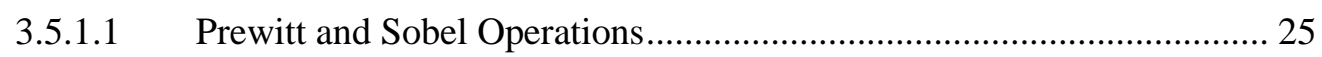

3.5.1.2 The Canny Edge Detection Method...................................................... 26

4. GABOR FILTERS FOR BLOOD VESSELS DETECTION IN RETINA........ 28

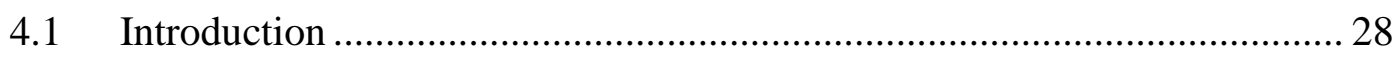

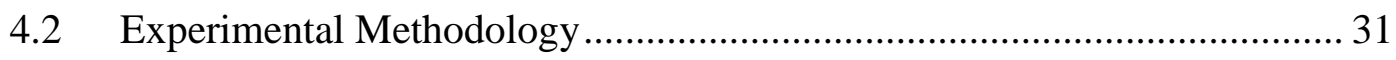

4.2.1 Sensitivity and Specificity …...................................................... 33

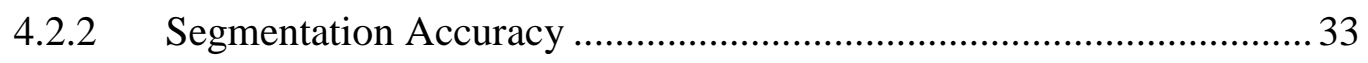

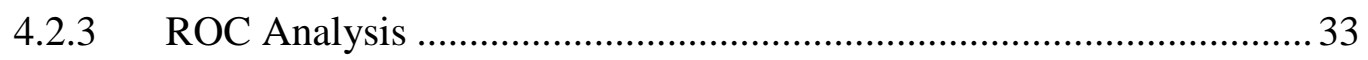

4.2.4 Thresholding (False Acceptance / False Rejection)............................ 34

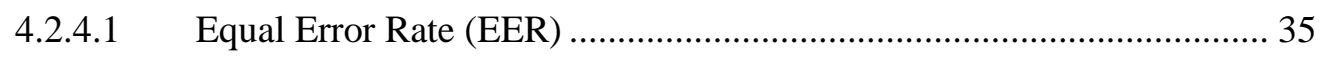

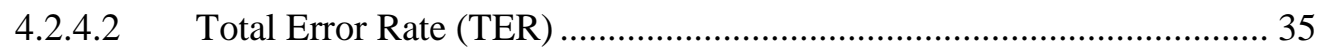

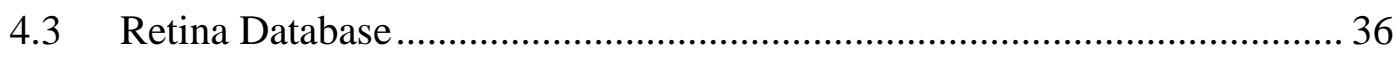

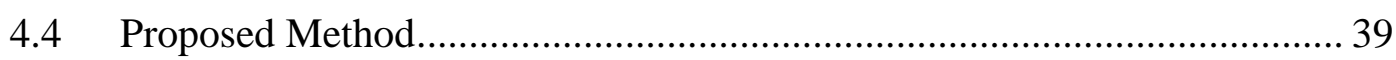




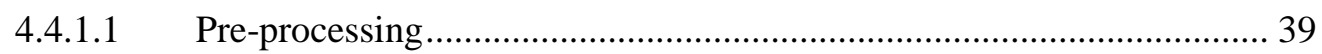

4.4.1.2 Gabor Filter for Detecting Oriented Patterns ...................................... 40

4.4.1.3 Maximum Response and Logarithm Normalization .............................. 42

4.4.2 Segmentation by Thresholding ................................................... 44

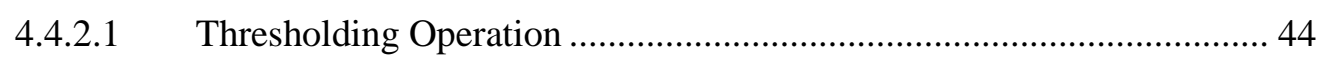

4.4.2.2 Morphological Erosion Operation ......................................................... 44

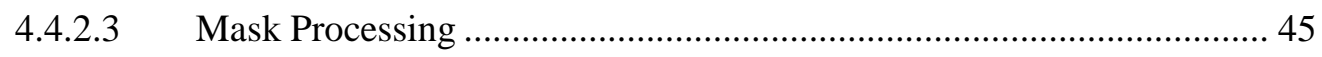

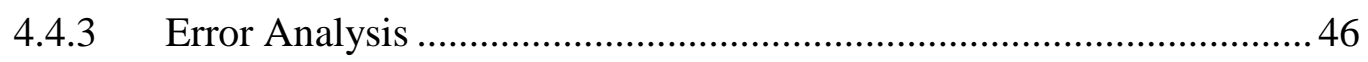

4.4.4 Mathematical Description of the Proposed Algorithms:...................... 48

5. SIMULATIONS, RESULTS AND DISCUSSIONS …................................. 51

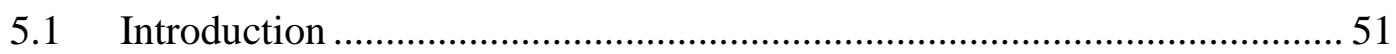

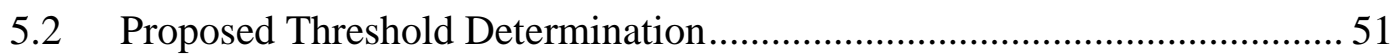

5.3 Analysis of Changing Number of Directions in Gabor Filters.................... 61

5.4 Thickness and Elongation Values in Gabor filter ..................................... 64

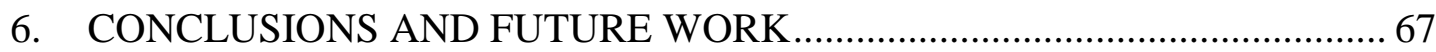

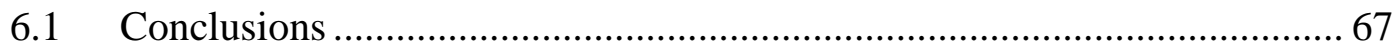

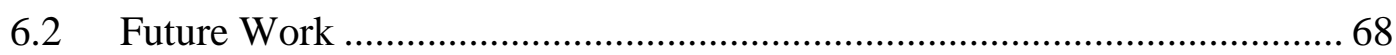

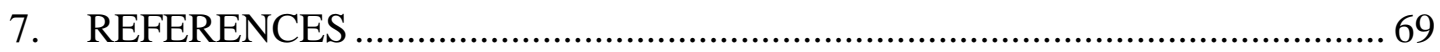




\section{LIST OF FIGURES}

Figure 2.1: Eey's Anatomy View with an Example of a Retinal Image 6

Figure 2.2: Loss of Central Vision due to Diabetic Retinopathy. (1) The Vision of a Healthy Patient. (2) The Vision of a Patient with the Retina Damaged in the Macula Region. ......................................................................... 8

Figure 2.3: A Patient Being Imaged with a Digital Fundus Camera [14]................... 9

Figure 2.4: Example of Ophthalmic Photography. (a) Fluoresce in Angiogram

(b) Digital Fundus Photograph [15] . 11

Figure 3.1: Window Function Frequency Response [28] ................................... 15

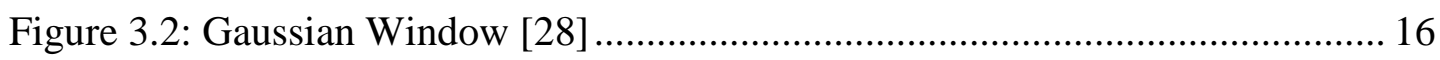

Figure 3.3: Gabor Filter Composition for 1D Signals: (a) Sinusoid, (b) A Gaussian Kernel, (c) The Corresponding to Gabor Filter [17] 18

Figure 3.4: Example of 2-D Gabor Filter. 18

Figure 3.5: The Real (a) and Imaginary Parts of a Complex Sinusoidal (b) [31]..... 19

Figure 3.6: A Gaussian Envelope [31]..... 20

Figure 3.7: (a) The Real and (b) Imaginary Parts of a Complex Gabor Function in Space Domain [31] 21

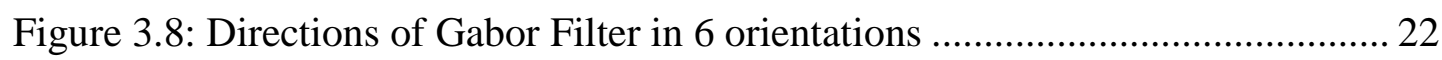

Figure 3.9: (a) Gabor Filter (b) Fourier of Gabor Filter ........................................ 23

Figure 3.10: Half-Magnitude Frequency …..................................................... 24

Figure 4.1: System Diagram of the Proposed Approach for Vessel Segmentation using Gabor Filters. 30 
Figure 4.2: Illustration of the Measure of Overlap between Two Segmentations where A Is Proposed Segmentation and B Is Manual Segmentation....... 32

Figure 4.3: Illustration of the ROC Curve for a Binary Result................................ 34

Figure 4.4: Equal Error Rate (EER) .................................................................... 35

Figure 4.5: Biometric Systems Are Rated on the Basis Performance Standards....... 36

Figure 4.6: (a) Image 01 of the Test Sets. (b) The Mask Delimiting the FOV. (c) The Manual Segmentation of the First. (d) The Second Observer [9] .......... 38

Figure 4.7: Appearance of Vessels in Different Color Channels. (a) Color Retinal Image. (b) Red Channel Image. (c) Green Channel Image. (d) Blue Channel Image[10] 40

Figure 4.8: (a) Image 07 Test Set (b) Input Intensity Image. Magnitude Response of 180 Gabor Filters over $0^{\circ}-180^{\circ}$ before Thresholding. (c) $\tau=4$ Pixels, $1=$ 2.5. (d) $\tau=8$ Pixels, $1=2.5$. 43

Figure 4.9: (a) The Image 07 Normalized by Threshold of 0.43. (b) The Mask Image 07 (c) Morphological Thinning Is Applied to (b). (d) The Area Around of Image Is Eliminated with Mask 46

Figure 4.10: (a) The Manual Image 07 Test Set. (b) Morphological Thinning Applied at the Boundaries (a). (c) The Segmented Output Image. (d) Comparison between Image (b) and Image (c) Containing Error Pixels. (e) The Red Pixels Corresponding to False Positives. (f) The Blue Pixels Corresponding to False Negatives. 48

Figure 5.1: The False Positive Ratio Corresponding to Threshold for Three Different Approaches 58

Figure 5.2: The True Positive Ratio Corresponding to Threshold for Three Different Approaches 59 
Figure 5.3: The Accuracy Corresponding to Threshold for Three Different Approaches 60

Figure5.4: The Specificity Corresponding to Threshold for Three Different Approaches 60

Figure 5.5: The True Positive Ratio Corresponding to Number of Direction in Image 19 of DRIVE Database 63

Figure 5.6: The Accuracy Corresponding to Number of Direction in Image 19 of DRIVE Database 63

Figure 5.7: The Area Under Curve Corresponding to Number of Direction in Image 19 of DRIVE Database 64

Figure 5.8: The Area Under Curve Corresponding to Thickness of Gabor Filter in Image 19 of DRIVE Database 65

Figure 5.9: The Area Under Curve Corresponding to Elongation of Gabor Filter in Image 19 of DRIVE Database 66 


\section{LIST OF TABLES}

Table 4.1: Relations between the Measurement Probabilities of the Manual Segmentation and Automatic Segmentation 31

Table 5.1: Performance of the Proposed Segmentation Method for 20 Images of DRIVE Database, Using Threshold Based on EER 53

Table 5.2: Performance of the Proposed Segmentation Method for 20 Images of DRIVE Database, Using Threshold Based on TER 54

Table 5.3: Performance of the Proposed Segmentation Method for 20 Images of DRIVE Database, Using Threshold Based on Maximum Accuracy ... 55

Table 5.4: Performance Accuracy, Specificity and Area Under Curve of the Retina Blood Vessels Achieved by Three Different Approaches 56

Table 5.5: Vessels Segmentation Accuracy of the Proposed Method and the Alternative Methods in the Literature 57

Table 5.6: Comparison of the Area Under Curve of Blood Vessels in the Retina Achieved by Different Methods 58

Table 5.7: Result of Changing Number of Direction in Gabor Filter for Image 19 of DRIVE Database According Approach 3 62

Table 5.8: Relation between Thickness and Elongation in Area Under Curve (AUC) 66 


\section{LIST OF SYMBOLS AND ABBREVIATIONS}

\begin{tabular}{|c|c|}
\hline $2-D$ & Two Dimension \\
\hline$(a, b)$ & Scale the two axis of the Gaussian envelop \\
\hline$p$ & Phase of the sinusoidal carrier \\
\hline$K$ & Scales the magnitude of the Gaussian envelop \\
\hline$\left(x_{0}, y_{0}\right)$ & Location of the peak of the Gaussian envelop \\
\hline$\left(u_{0}, v_{0}\right)$ & Spatial frequencies of the sinusoidal carrier in Cartesian \\
\hline$\ominus$ & morphological erosion operation \\
\hline$\Theta$ & Rotation angle of the Gaussian envelop \\
\hline AUC & Area Under a Curve \\
\hline EER & Equal Error Rate \\
\hline FN & False Negative \\
\hline FOV & Field Of View \\
\hline FP & False Positive \\
\hline FNR & False-Negative Ratio \\
\hline FPR & False-Positive Ratio \\
\hline ICG & Indo cyanine Green \\
\hline IEEE & Institute of Electrical and Electronics Engineers \\
\hline ROC & Receiver Operating Characteristic \\
\hline SE & Sensitivity \\
\hline SP & Specificity \\
\hline TER & Total Error Rate \\
\hline $\mathrm{TN}$ & True Negative \\
\hline TNR & True-Negative Ratio \\
\hline
\end{tabular}


TPR

True-Positive Ratio

xvii 


\section{Chapter 1}

\section{INTRODUCTION}

\subsection{Motivation}

Currently, the advances on digital imaging systems offer very high-resolution images that can be used in many applications from several areas like medicine. The transmission of this kind of images makes them suitable to integrate their use into large scalable systems. Moreover, these images can be improved and even be subjected to techniques to perform an objective quantitative and qualitative analysis.

As for the medical image processing is concerned, a lot of research has been focused on the computation of accurate geometric models of anatomic structures from medical images in order to use the potential of automated applications for early disease diagnosis. The detection of blood vessels is an important step in applications of image processing in ophthalmology [1]. Retinal vascular segmentation techniques utilize the contrast existing between the retinal blood vessels and the surrounding background. The cross-sectional gray-level profile of a typical vessel conforms to a Gaussian shape. The vasculature is piecewise linear and may be represented by a series of connected line segments. Five main techniques used to segment the vasculature in retinal images are matched filters [2], Gabor filters [3], vessel tracking [4], neural networks [5], and morphological processing [6]. The motivation for the use of Gabor filters in texture analysis is double folded. First, it is believed that simple cells in the visual cortex can be modeled by Gabor functions, and that the 
Gabor scheme provides a suitable representation for visual information in the combined spatial-frequency [7]. Second, the Gabor representation has been shown to be optimal in the sense of minimizing the joint two-dimensional uncertainty in the combined frequency- space domain [8]. The blood vessel detection in retinal is essential for most applications of image analysis in ophthalmology which could assist in the monitoring of the effects of some diseases such as diabetes and hypertension on the visual system. The methods include the design of a bank of Gabor filters can be used to detect the changes in the shape, width, and tortuosity of the vessels.

\subsection{Thesis Objective}

In this thesis we propose to use the Gabor filters for detection of vessels in retinal images. Filter responses are combined and thresholded for segmentation. In this context the main objectives are:

- Using Gabor filters for efficient retina vessels detection. Study multiple direction and scales for robust detection.

- Use thresholding for segmentation. Furthermore, develop a systematic way of deciding an efficient threshold value.

- Apply Receiver Operating Characteristic (ROC) analyses for efficient performance measurements including True-Positive Ratio (TPR), FalsePositive Ratio (FPR), sensitivity, specificity, accuracy, Equal Error Rate (EER) and also Total Error Rate (TER).

- Compare the generated results with the alternative results of the other methods available in the literature. 


\subsection{Thesis Contributions}

In this thesis, we propose to use Gabor filter for the detection of vessels in retina images. This involves two main steps. The first is to capture edges by using a bank of 180 Gabor filters with $1^{\circ}$ of separation in their orientation. The second step involves with different image processing methods, which consists of logarithm normalization, morphological operation and mask processing. Also we are proposing a systematic way of thresholding for improved segmentation performance. Using retina images contained within a standard image database, we show that generated results are comparable by the other state-of the-art results in the relevant literature. We obtained human retinal images from publicly available databases. The source of fundus images used to test the segmentation is the DRIVE (Digital Retinal Image for Vessel Extraction) database [9]. In all cases, color images were converted to intensity image by extracting the green channel information, because the green channel exhibits the best contrast for vessel detection [10].

\subsection{Thesis Organization}

Chapter 2 introduces the background of medical images. It includes anatomy of the ocular fundus and the landmark retinal structures. Then Eye complications and detection of blood vessels in retina are described. Chapter 3 gives a complete idea about the fundamentals of Gabor filters. It also contains the details of the various parameters upon which the working of the Gabor filters depends. Chapter 4 describes acquisition of retinal image and pre-processing methodologies. The specifications of the retinal images that are used in this work are discussed and also present the Gabor filter for detection of retinal vessels. The properties of the vessels and how they are used to design the Gabor filter is described. A set of Gabor filters oriented along 
different directions are used to enhance the vessels. It is followed by segmentation of vessel pixels using a suitable threshold. Details of retinal images obtained from standard databases are mentioned. The performance of the proposed method on DRIVE [9] database is stated. In Chapter 5 the performance of the proposed method is analyzed and the comparison of the simulation results with other vessel segmentation approaches is given. Chapter 6 presents thesis conclusions on the basis of analysis and discussion and highlights the contributions of this work. It also includes scope for improvement and future direction of research. 


\section{Chapter 2}

\section{USING RETINAL BLOOD VESSELS ANAYSIS FOR DISEASE DIAGNOSIS}

\subsection{Introduction}

As for the medical image processing is concerned, a lot of research has been focused on the computation of accurate geometric models of anatomic structures from medical images in order to exploit the potential of automated applications for early disease diagnosis, security tasks, model bone reconstruction, and so on. Medical imaging allows scientists and physicians to understand potentially lifesaving information without doing anything harmful to the patient. It has been used as research tool to surgical planning and simulation and for tracking the progress of diseases. Diabetes and its associated complications have been identified as significant growing public health problems. The worldwide prevalence of diabetes was estimated to be $2.8 \%$ in 2000 , projected to $4.4 \%$ in 2030 [11]. Retinal vessels segmentation is a primary step towards the automated analysis of the retina, to be applied in a screening tool for early detection of diabetic retinopathy.

In this chapter, blood vessels are analyzed to diagnose diseases of the retina vessels. First, a brief explanation about the anatomy of eye and retinal cameras is made. Then, the different types of medical images state of the art in the retinal vessel extraction are presented. Finally, the retinal imaging and vessel properties are discussed respectively. 


\subsection{Anatomy of Eye}

Vision is the most used of the five senses in the human body. We rely on our eyes to provide most of the data we detect about the world, so much so, that a significant portion of the brain is devoted entirely to visual processing. The eye is often compared to a camera because of the way it processes light into data understandable by the brain. Both have lenses to focus the incoming light. Whereas the eye uses a Specialist layer of cells that, called the retina, camera uses the film to create a picture. However the eye's ability of focus on a wide range of objects having different sizes, contrast at a high speed and luminosity are more powerful than those of current cameras.

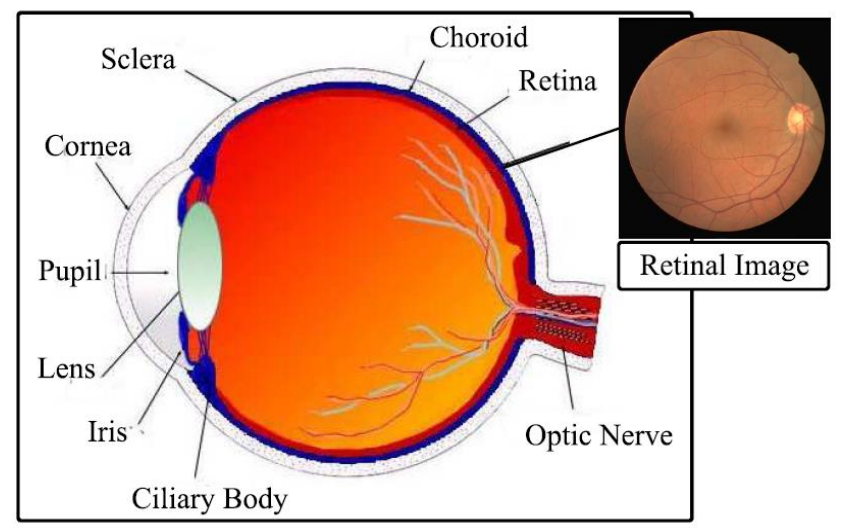

Figure 2.1: Eey's Anatomy View with an Example of a Retinal Image

The light sensitive inner layer of the eye, which receives images formed by the lens and transmits them through the optic nerve to the brain, is a multi-layered sensory tissue that lies on the back of the eye. 
It contains millions of photoreceptors that capture light rays and convert them into electrical impulses. These impulses travel along the optic nerve to the brain where they are converted into images. A retinal fundus image given in Figure 2.1 is defined as the interior lining of the eyeball, including the retina, optic disk, the retinal vessel tree and the macula (the small spot in the retina where vision is keenest). The fundus is the portion of the inner eye that can be seen during an eye examination by looking through the pupil. Fundus retinal images are used to study the main vessel features and the vascular structure in many tools applications.

There are two types of photoreceptors in the retina: rods and cones, named after their shape. Rods are sensitive cells to changes in contrast even at low light levels, therefore able to detect movement, but they are inexact insensitive and inexact to color. They are concentrated in the periphery of the retina and used for scotopia (night vision). Cones, on the other hand, are sensitive cells capable to detecting color at high precision. They are located in the macula and used for photopic vision (day vision). The very central part of the macula is fovea, which is where the human eye is able to distinguish visual details at its best. Whereas loss of peripheral vision may go unnoticed for some time, damage to the macula will result in loss of central vision, which has serious effects as it is illustrated in Figure 2.2 [12]. 


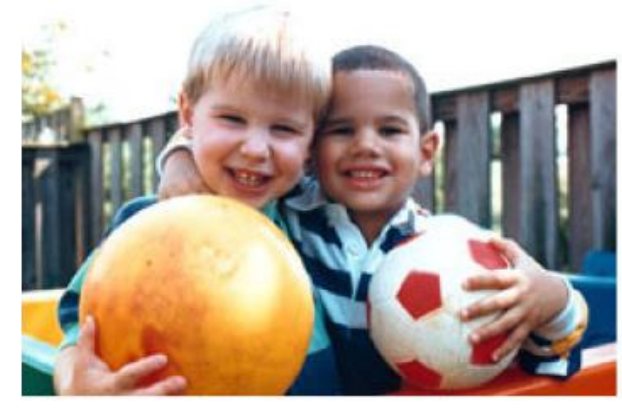

(a)

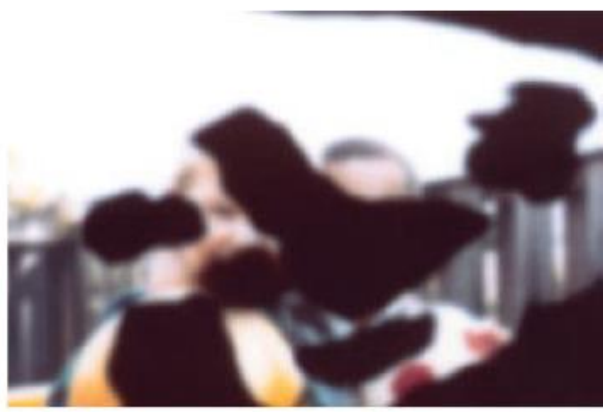

(b)

Figure 2.2: Loss of Central Vision due to Diabetic Retinopathy. (1) The Vision of a Healthy Patient. (2) The Vision of a Patient with the Retina Damaged in the Macula Region.

All the photoreceptors are connected to the brain through a dense network of roughly 1.2 million of nerves [13]. All nerves leave the eye in a unique bundle in the optic nerve. In the retina there is a blind spot which is a result of the absence of retina photoreceptors where the optic nerve leaves the eye.

Early detection and treatment of various types of retinopathy are crucial to avoid preventable vision loss. Imaging of the retina began with human retinal photography. Fluorescein angiography is an imaging method used in the diagnosis and management of retinal diseases [14]. Fluorescein angiography needs to inject of fluorescein into the blood. However, with the recent progress in communications technology and information, developed techniques for digital photography of the retina have been developed. Digital fundus images of the retina are now an essential means to document and diagnose various eye diseases in clinics, and they are widely used to screen large populations for systemic diseases, such as diabetes, arteriosclerosis, and hypertension. Figure 2.3 indicates a patient being imaged with a digital fundus camera. 

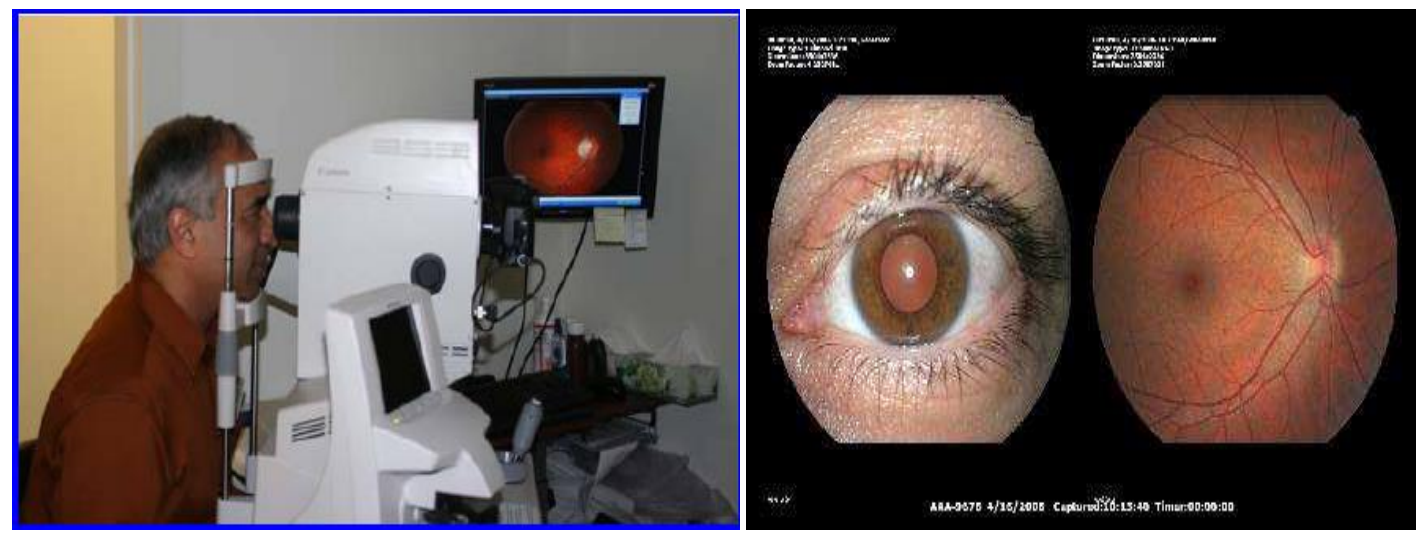

Figure 2.3: A Patient Being Imaged with a Digital Fundus Camera [14]

The obvious advantages of digital images of the retina are the permanence of the record, the facilities for observer control, and the opportunity for precise comparison of the states of the retinal fundus at particular intervals of time. Additional advantages are the possibilities for precise measurement of the diameter of vessels and for observation of blood flow.

In this thesis, digital color fundus and red free images (also called retinal images) have been selected because the method to get them is the least intrusive. The obvious advantages of digital images of the retina are the permanence of the record, the facilities for observer control, and the opportunity for precise comparison of the states of the retinal fundus at particular intervals of time. Additional advantages are the possibilities for precise measurement of the diameter of vessels and for observation of blood flow. 


\subsection{Retinal Imaging}

Ophthalmic photography is a form of medical imaging dedicated to the study and treatment of disorders of the eye. There are two common procedures to perform such photography: (a) angiography and (b) fundus photography. Angiography is the imaging of vessels, and the resulting pictures are angiograms. Angiography of the retina of the eye provides the injection of a small amount of dye into a vein in the patient's arm. The dye travels through the bloodstream and using special cameras is photographed. Type of this procedure, fluorescein angiogram is shown in Figure 2.4.

Alternatively, when performing ophthalmic fundus photography for diagnostic purposes, the pupil is dilated with eye drops and a special camera called a fundus camera is used to focus on the fundus. The resulting images are detailed and revealing, showing the optic nerve through which visual 'signals' are transmitted to the brain and the retinal vessels which supply nutrition and oxygen to the tissue. A digital fundus photograph is shown in Figure 2.4 (b), where the contrast between the blood vessels and retinal background is not as good as a fluorescein angiogram. Fundus photograph usually to capturing blood vessels in retinal images uses a green filter (red-free). In the fundus photograph green light is absorbed by blood and appeared darker color than the background and the retinal nerve fiber layer. 


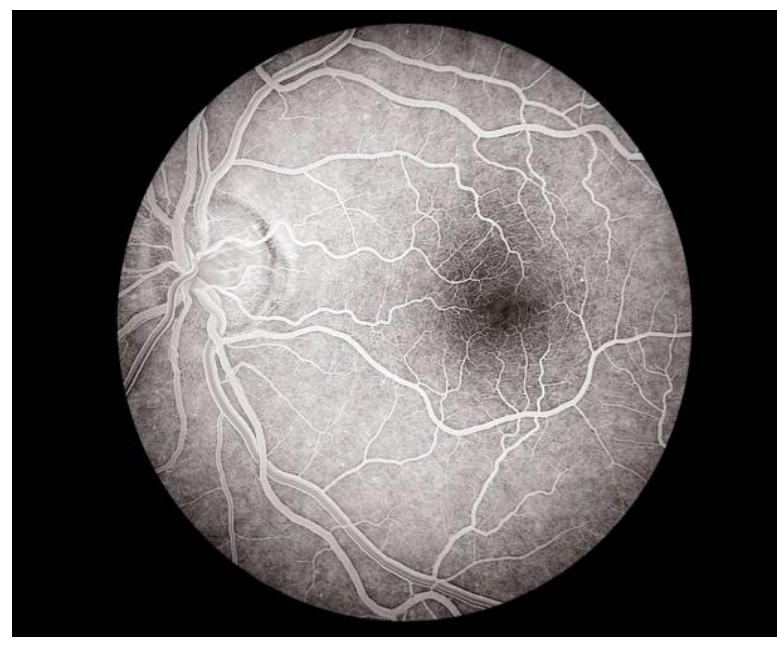

(a)

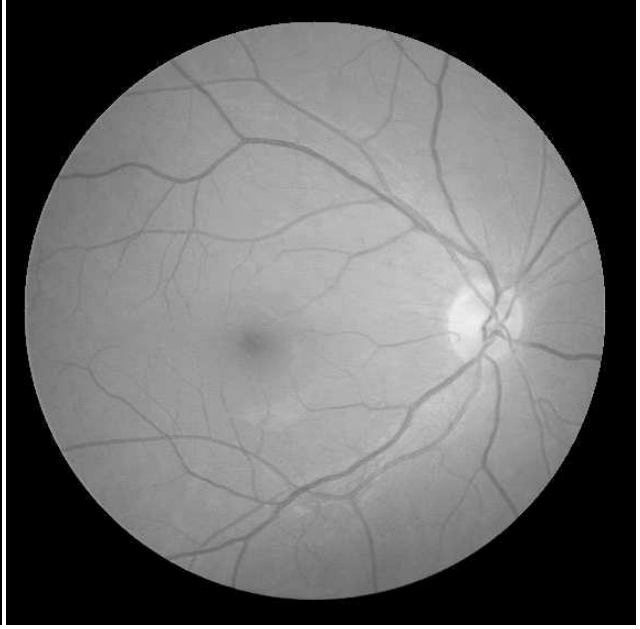

(b)

Figure 2.4: Example of Ophthalmic Photography. (a) Fluoresce in Angiogram (b) Digital Fundus Photograph [15]

However fluorescein angiography due to high contrast between the blood vessel and background retinal layer can detect and quantify changes in the blood vessels more accurately than fundus photography, but it sometimes is unsuitable for certain people because of allergic reactions. Therefore fundus photography is more widely used in clinics. Despite the contrast between the blood vessels and retinal background tends to be poor in fundus photography. Thus accurate vessel segmentation on fundus photography is harder than from other photographic procedures.

\subsection{Vessel Properties and their Applications}

The vessels usually have a limited curvature and are often approximated by piecewise linear segments. Even though the width of the vessels decreases as they move radially outward from the optic disc, the change is gradual one. The widths of the vessels are found to lie within a range of $40-200 \mu \mathrm{m}$ in a standard retinal image. These properties were exploited and a matched filter was designed to segment the vessels. The segmentation and analysis of retinal vasculature form an essential part 
of several practical applications such as detection of hypertension, diabetes, stroke and cardiovascular diseases. In case of ophthalmologic conditions, the segmentation and measurement of the retinal vessels is of primary interest in the diagnosis and treatment of a diabetic retinopathy that directly affect the morphology of the retinal vessel tree. Also the accurate segmentation of the retinal blood vessels is often an essential prerequisite step in the automated analysis of retina for characterizing the detected lesions and in identifying false positives [14] . 


\section{Chapter 3}

\section{GABOR FILTERS AND THEIR APPLICATIONS}

\subsection{Introduction}

Gaussian functions modulated by sine waves are known in signal and image processing as Gabor functions, has introduced by Dennis Gabor in 1946 [16]. However, these functions have played an effective role before 1946 in harmonic analysis and context of quantum mechanics. Gabor has shown that the representation of signals using the Gaussian functions modulated by complex exponentials is optimal in the sense of minimizing the joint uncertainty in the combined timefrequency space [18]. Thus, these functions provide the best trade-off between time resolution and frequency resolution. Then Gabor was extended to two dimensions by Daugman [23]. Several years of research of the Human Visual System provided a model of the organization of the visual cortex which culminated in the important discovery of Marcelja [18] and Daugman [23] that simple cells in the visual cortex can be modeled by Gabor functions. An analytical method suitable for the calculation of the Gabor expansion coefficients was presented by Bastiaans [19] for the onedimensional case and extended to two-dimensions by Porat and Zeevi [20] who have shown that the Gabor scheme provides a suitable representation for visual information in the combined frequency-position space. These findings have stimulated a great deal of interest in the computer vision and image processing communities, as in these communities there is an everlasting quest to provide a 
mathematical model for the human vision and use this model for applications. This is because it is often assumed that the human visual system has evolved to process visual data optimally, i.e. best matched to the structure of our environment. Ever since, the original work of Gabor has been extended and applied in representation, enhancement, restoration, segmentation and compression of images, all the way up to the ultimate tasks of recognition and interpretation [21]. Because the Gabor functions have enjoyed a great deal of interest in the wavelets community, the framework of Gabor wavelets has become very popular. Gabor wavelets, characterized by their optimal (minimal), inversely-related effective widths in position and frequency, have become important means for many image processing applications.

In this chapter first, we give a short introduction to Gaussian functions as a part of Gabor function. Then, we proceed with a deeper consideration of Gabor filters It also contains the details of the various types of Gabor filters. At the end the application of this function has introduced.

\subsection{Window Function}

A filter can be mathematically represented as a window function which can then be applied to a signal for processing. A window function is ideally a function that is constant inside a specified region and zero outside it. It is used for analyzing a certain region and rejecting the undesired parts. As for the practical cases, a window function is suitable inside the desired region and decays fast outside, with an emphasis on trying to achieve the highest compatibility with the ideal case. The width of the main lobe determines the ability to resolve comparable strength signals, 
whereas the peak levels of the side lobes determine the ability to resolve disparate strength signals. Window function frequency response is represented in Figure 3.1. The Gaussian window is a typical window function used in this project work.

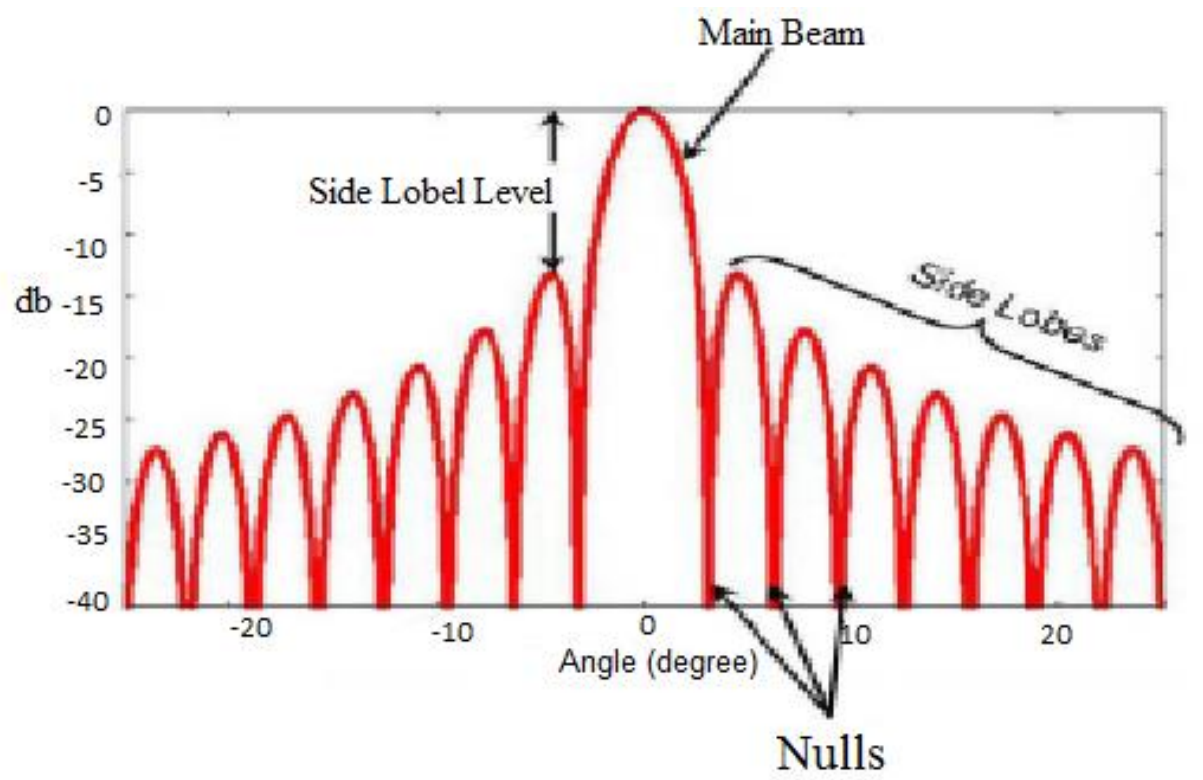

Figure 3.1: Window Function Frequency Response [28]

\subsubsection{Gaussian Window}

The Gaussian function is selected because of some unique characteristics as the Fourier transform and the derivative of a Gaussian function are both Gaussian functions. This property is greatly helpful in our time-frequency analysis. Gaussian function also has a reasonable capability to resolve disparate strength signals, as it possesses the properties of a window function. A Gaussian window and its frequency response are given in Figure 3.2. 

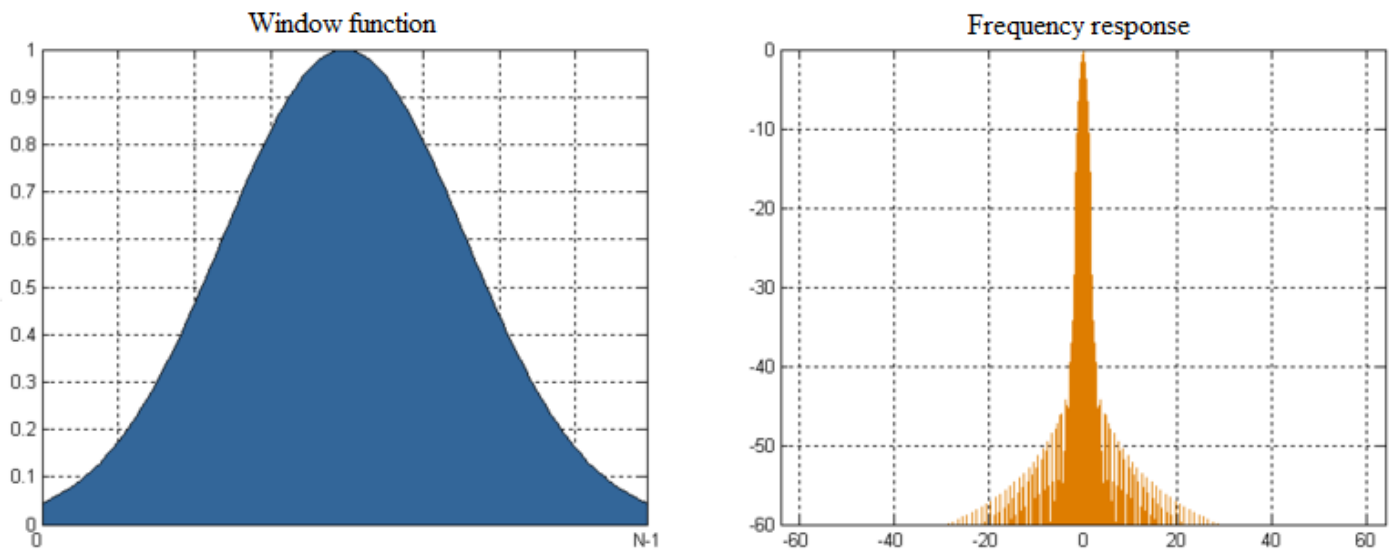

Figure 3.2: Gaussian Window [28]

\subsection{Gabor Filters}

A Gabor filter is a linear filter whose impulse response is defined by a harmonic function multiplied by a Gaussian function. Because of the multiplicationconvolution property (Convolution theorem), the Fourier transform of a Gabor filter's impulse response is the convolution of the Fourier transform of the harmonic function and the Fourier transform of the Gaussian function.

Gabor filters are directly related to Gabor wavelets, since they can be designed for number of dilations and rotations. However, in general, expansion is not applied for Gabor wavelets, since this requires computation of biorthogonal wavelets, which may be very time-consuming. Therefore, usually, a filter bank consisting of Gabor filters with various scales and rotations is created. The filters are convolved with the signal, resulting in a so-called Gabor space. This process is closely related to processes in the primary visual cortex. The Gabor space is very useful in e.g., image processing applications such as retina identification, edge detection and texture segmentation. Relations between activations for a specific spatial location are very 
distinctive between objects in an image. Furthermore, important activations can be extracted from the Gabor space in order to create a sparse object representation.

\subsubsection{One-dimensional Gabor Filter}

The 1-D Gabor Filter was first introduced by D. Gabor in 1946. A Gabor filter can be viewed as a sinusoidal plane of particular frequency and orientation, modulated by a Gaussian envelope [29].

Whereas $\mathrm{s}(\mathrm{x}, \mathrm{y})$ is a Complex sinusoid and $\mathrm{g}(\mathrm{x}, \mathrm{y})$ is 2-D Gaussian shaped function, defined as envelope:

$$
\begin{gathered}
h(x, y)=s(x, y) g(x, y) \\
s(x, y)=e^{-2 \pi j\left(u_{0} x+v_{0} y\right)} \\
g(x, y)=\frac{1}{\sqrt{2 \pi \sigma}} e^{-\frac{1}{2}\left(\frac{x^{2}}{\sigma_{x}{ }^{2}}+\frac{y^{2}}{\sigma_{y}{ }^{2}}\right)}
\end{gathered}
$$

Where $\left(\mathrm{u}_{0}, \mathrm{v}_{0}\right)$ are spatial frequency and $\sigma_{\mathrm{x}}$ and $\sigma_{\mathrm{y}}$ are standard deviation in $\mathrm{x}$ and $\mathrm{y}$ directions, respectively. P defines the phase of the sinusoidal.

A simple way to view the responses of Gabor filters have been devised in one dimension. These filters responses are summarized in a static one-dimensional graph and provide an introduction to our approach for two dimensional filters. Figure 3.3 illustrates sinusoid, Gaussian Kernel corresponding to one dimensional Gabor filter. 


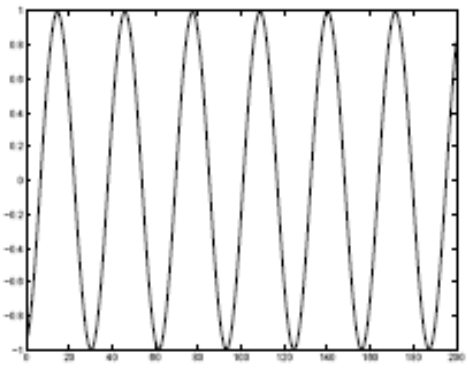

(a)

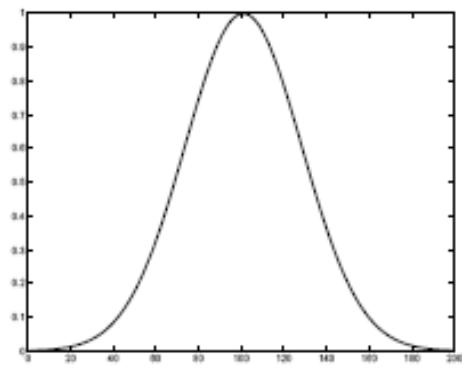

(b)

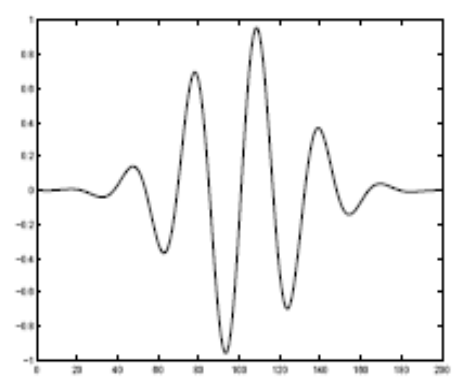

(c)

Figure 3.3: Gabor Filter Composition for 1D Signals: (a) Sinusoid, (b) A Gaussian Kernel, (c) The Corresponding to Gabor Filter [17]

\subsubsection{Two- dimensional Gabor filter}

The filter collection originally proposed by Gabor was extended to the twodimensional case by Daugman [29]. A Gabor filter centered at the 2D frequency coordinates is shown in Figure 3.4.

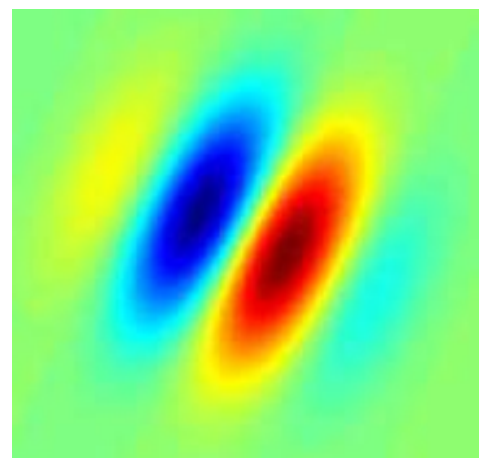

Figure 3.4: Example of 2-D Gabor Filter

$$
g(x, y)=s(x, y) w_{r}(x, y)
$$

Where $\mathrm{S}(\mathrm{x}, \mathrm{y})$ is a complex sinusoidal, known as the carrier and the complex sinusoidal is introduced as follow:

$$
S(x, y)=e^{\left(j\left(2 \pi\left(u_{0} x+v_{0} y\right)+p\right)\right)}
$$




$$
\begin{aligned}
& \left.\operatorname{Re}(s(x, y))=\cos \left(2 \pi\left(u_{0} x+v_{0} y\right)+p\right)\right) \\
& \left.\operatorname{Im}(s(x, y))=\sin \left(2 \pi\left(u_{0} x+v_{0} y\right)+p\right)\right)
\end{aligned}
$$

The sinusoid has a real component and an imaginary component that have been indicated in Figure 3.5. The Images are $128 \times 128$ Pixels with Parameters are as Follows: $\mathrm{u}_{0}=\mathrm{v}_{0}=\frac{1}{80} ; \mathrm{p}=0$.

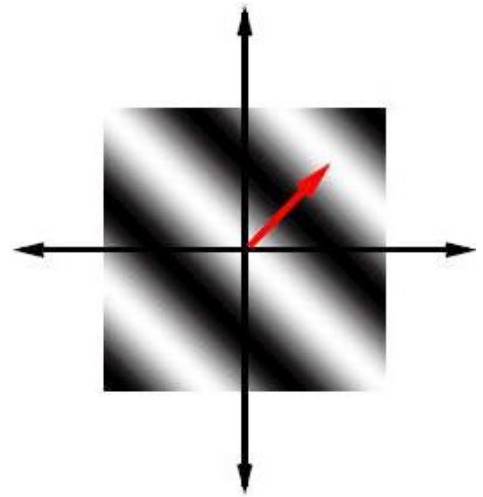

(a)

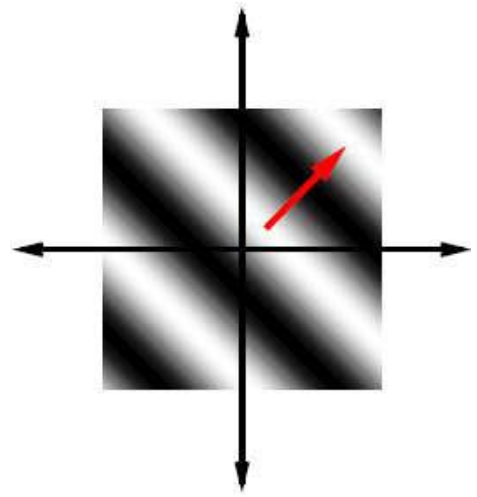

(b)

Figure 3.5: The Real (a) and Imaginary Parts of a Complex Sinusoidal (b) [31]

According this representation, the complex sinusoid can be written as:

$$
S(x, y)=e^{\left(j\left(2 \pi F_{0}\left(x \cos w_{0}+y \sin w_{0}\right)+p\right)\right)}
$$

$\mathrm{w}_{\mathrm{r}}(\mathrm{x}, \mathrm{y})$ is a 2-D Gaussian-shaped function, known as envelop.

$$
\mathrm{w}_{\mathrm{r}}(\mathrm{x}, \mathrm{y})=\mathrm{K} \mathrm{e}^{\left(-\pi\left(\left(\mathrm{a}^{2}\left(\mathrm{x}-\mathrm{x}_{0}\right)_{\mathrm{r}}^{2}+\mathrm{b}^{2}\left(\mathrm{y}-\mathrm{y}_{0}\right)_{\mathrm{r}}^{2}\right)\right)\right.}
$$

In this formula $\mathrm{K}$ is Scales the magnitude of the Gaussian envelop, $\left(\mathrm{x}_{0}, \mathrm{y}_{0}\right)$ is the peak of the function, $a, b$ are scaling parameters of the Gaussian, and the $r$ subscript stands for a rotation operation. This allows us to have Gabor filters with different 
orientations. A Gaussian envelope in frequency domain has indicated in Figure 3.6. The complexity of convolution depends directly on the size of the convolution mask.

$$
\begin{aligned}
& \left(x-x_{0}\right)_{r}=\left(x-x_{0}\right) \cos \theta+\left(y-y_{0}\right) \sin \theta \\
& \left(y-y_{0}\right)_{r}=-\left(x-x_{0}\right) \sin \theta+\left(y-y_{0}\right) \cos \theta
\end{aligned}
$$

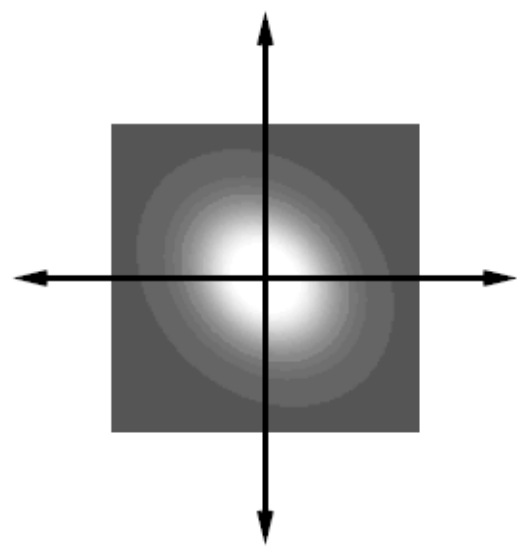

Figure 3.6: A Gaussian Envelope [31].

\subsection{The Complex Gabor Function in Space Domain:}

The complex Gabor filter in space domain can be written as follow:

$$
g(x, y)=\mathrm{ke}^{\left(-\pi\left(\mathrm{a}^{2}\left(\mathrm{x}-\mathrm{x}_{0}\right)_{\mathrm{r}}^{2}+\mathrm{b}^{2}\left(\mathrm{y}-\mathrm{y}_{0}\right)_{\mathrm{r}}^{2}\right)\right) \exp \left(\mathrm{j}\left(2 \pi\left(\mathrm{u}_{0} \mathrm{x}+\mathrm{v}_{0} \mathrm{y}\right) \mathrm{p}\right)\right)}
$$

Therefore general form of 2-D Gabor filter has defined with 9 parameters:

$\mathrm{k} \quad$ : Scales the magnitude of the Gaussian envelop

$(a, b) \quad$ : Scale the two axis of the Gaussian envelop

$\theta \quad$ : Rotation angle of the Gaussian envelop

$\left(\mathrm{x}_{0}, \mathrm{y}_{0}\right)$ : Location of the peak of the Gaussian envelop

$\left(\mathrm{u}_{0}, \mathrm{v}_{0}\right)$ : Spatial frequencies of the sinusoidal carrier in Cartesian

P : Phase of the sinusoidal carrier 
The real and imaginary parts of complex Gabor function in space domain are represented in Figure 3.7.

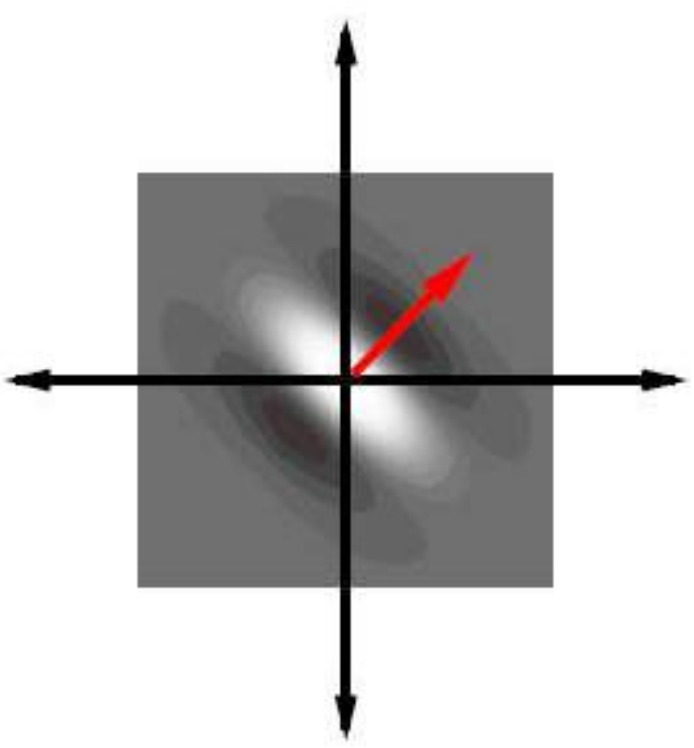

(a)

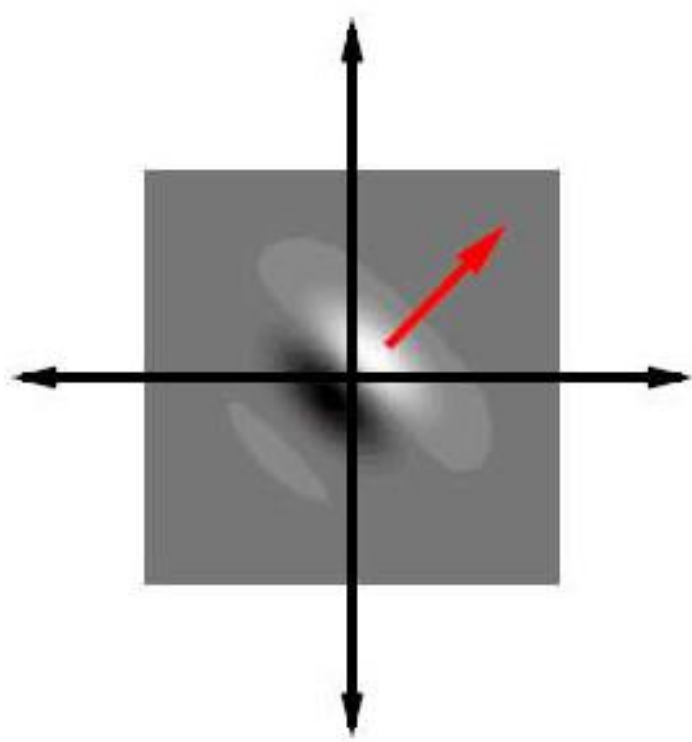

(b)

Figure 3.7: (a) The Real and (b) Imaginary Parts of a Complex Gabor Function in Space Domain [31]

$\left(u_{0}, v_{0}\right)$ Spatial frequencies of the sinusoidal carrier in Cartesian coordinate can be defined in polar coordinates as $\left(F_{0}, \varphi_{0}\right)$.

$$
\begin{gathered}
\mathrm{F}_{0}=\sqrt{\mathrm{u}_{0}^{2}+\mathrm{v}_{0}^{2}} \\
\varphi_{0}=\tan ^{-1} \frac{\mathrm{v}_{0}}{\mathrm{u}_{0}}
\end{gathered}
$$

i.e.

$$
\begin{aligned}
& \mathrm{u}_{0}=\mathrm{F}_{0} \cos \varphi_{0} \\
& \mathrm{v}_{0}=\mathrm{F}_{0} \sin \varphi_{0}
\end{aligned}
$$

Accordingly, the Gabor function in polar coordinates is:

$$
g(x, y)=\mathrm{k}^{\left.\left(\mathrm{a}^{2}\left(\mathrm{x}-\mathrm{x}_{0}\right)_{\mathrm{r}}^{2}+\mathrm{b}^{2}\left(\mathrm{y}-\mathrm{y}_{0}\right)_{\mathrm{r}}^{2}\right)\right)} * \mathrm{e}^{\left(\mathrm{j}\left(2 \pi \mathrm{F}_{0}\left(\mathrm{x} \cos \varphi_{0}+\mathrm{y} \sin \varphi_{0}\right)+\mathrm{p}\right)\right)}
$$


The filter applies to the image, emphasizing edges along the rotation of the Gaussian envelope.

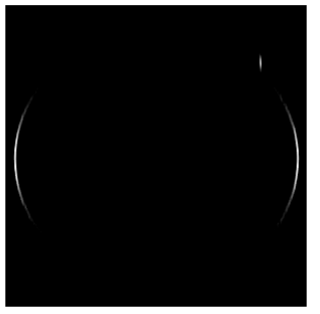

$0^{\circ}$
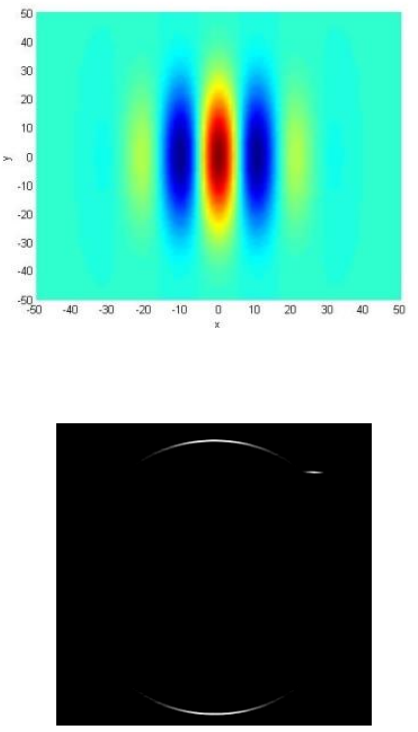

$90^{\circ}$

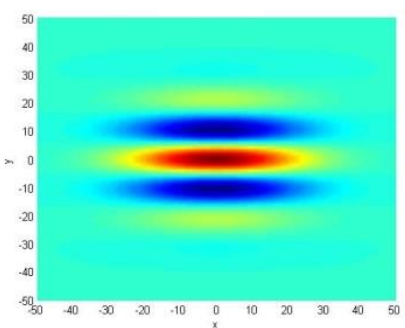

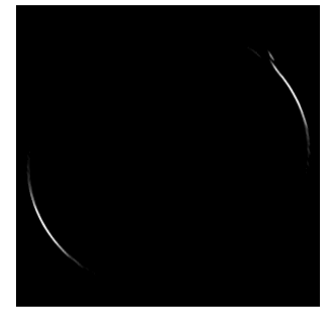

$30^{\circ}$
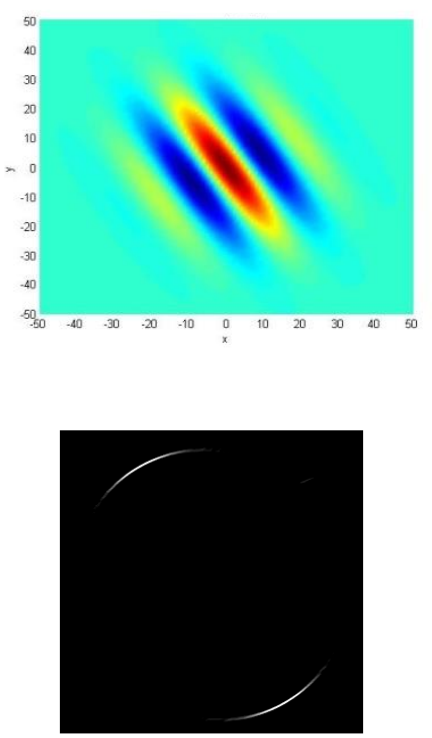

$120^{\circ}$

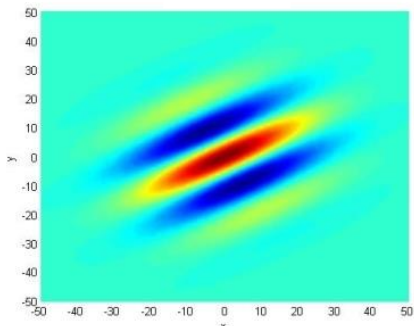

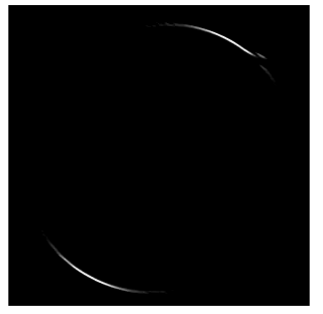

$60^{\circ}$
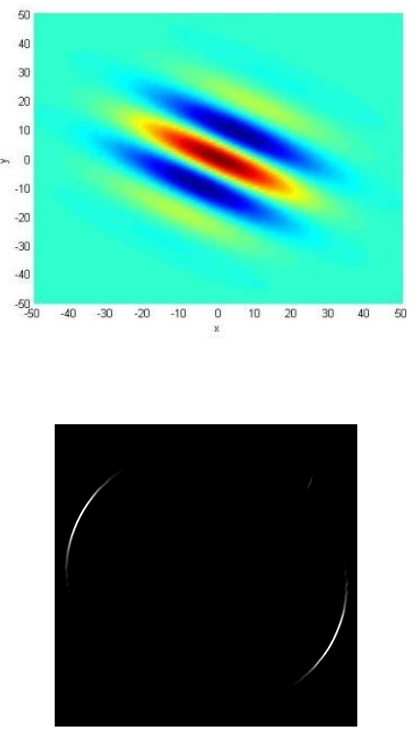

$150^{\circ}$

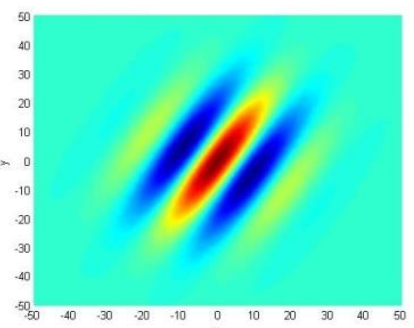

Figure 3.8: Directions of Gabor Filter in 6 orientations

In Figure 3.8 several examples of applying different orientations $\left(0^{\circ}, 30^{\circ}, 60^{\circ}, 90^{\circ}, 120^{\circ}\right.$, and $\left.150^{\circ}\right)$ of the Gabor function to an image are presented.

So, the Fourier transform of the Gabor function defined as follow: 


$$
G(u, v)=\frac{k}{a b} \mathrm{e}^{\left(j\left(-2 \pi\left(x_{0}\left(\mathrm{u}-u_{0}\right)+y_{0}\left(v-v_{0}\right)\right)+\mathrm{p}\right)\right)} * \mathrm{e}^{\left(-\pi\left(\frac{\left(\mathrm{u}-u_{0}\right)_{\mathrm{r}}^{2}}{a^{2}}+\frac{\left(v-v_{0}\right)_{\mathrm{r}}^{2}}{b^{2}}\right)\right)}
$$

Or in polar coordinates,

Magnitude

$$
g(u, v) \frac{k}{a b} e^{\left(-\pi\left(\frac{\left(u-u_{0}\right)_{r}^{2}}{a^{2}}+\frac{\left(v-v_{0}\right)_{r}^{2}}{b^{2}}\right)\right.}
$$

Phase

$$
g(u, v)=-2 \pi\left(x_{0}\left(u-u_{0}\right)+y_{0}\left(v-v_{0}\right)+p\right.
$$

The Gabor filter and its Fourier transform are shown in Figure 3.9.

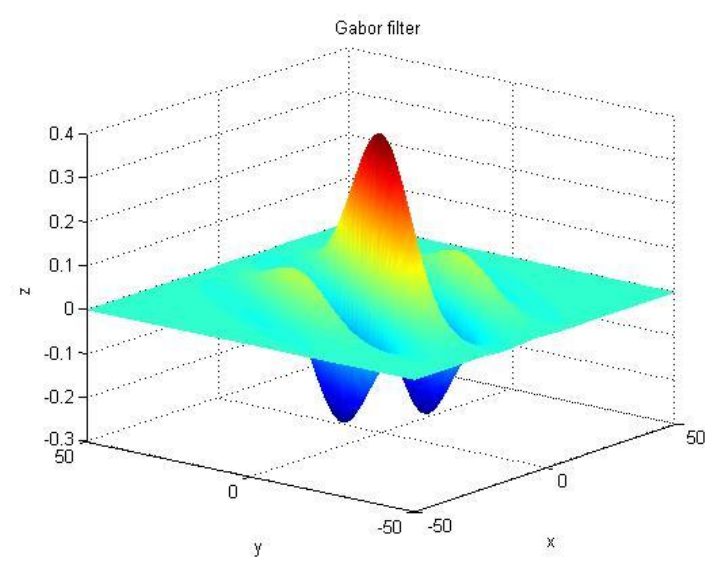

(a)

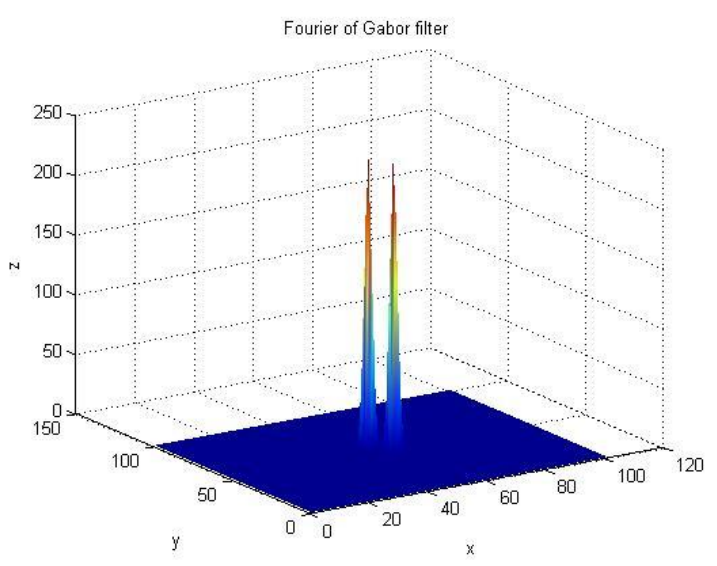

(b)

Figure 3.9: (a) Gabor Filter (b) Fourier of Gabor Filter

\subsubsection{Half-magnitude Profile}

The region of points, in frequency domain, with magnitude equal one- half the peak magnitude can be obtained as follows. Since the peak value is obtained for $(u, v)=$ $\left(u_{0}, v_{0}\right)$, and the peak magnitude is $\frac{K}{a b}$, we just need to find the set of points $(u, v)$ with magnitude $\frac{K}{2 a b}$. 


$$
\frac{\mathrm{K}}{2 \mathrm{ab}}=\frac{\mathrm{K}}{\mathrm{ab}} \mathrm{e}^{\left(-\pi\left(\frac{\left(\mathrm{u}-\mathrm{u}_{0}\right)_{\mathrm{r}}^{2}}{\mathrm{a}^{2}}+\frac{\left(\mathrm{v}-\mathrm{v}_{0}\right)_{\mathrm{r}}^{2}}{\mathrm{~b}^{2}}\right)\right)}
$$

or

$$
-\log 2=-\pi\left(\frac{\left(\mathrm{u}-\mathrm{u}_{0}\right)_{\mathrm{r}}^{2}}{\mathrm{a}^{2}}+\frac{\left(\mathrm{v}-\mathrm{v}_{0}\right)_{\mathrm{r}}^{2}}{\mathrm{~b}^{2}}\right)
$$

Here we consider c such as:

$$
\begin{gathered}
\mathrm{C}=\sqrt{\frac{\log 2}{\pi}} \cong 0.5 \\
{\left[\frac{\left(\mathrm{u}-\mathrm{u}_{0}\right)_{\mathrm{r}}}{\mathrm{aC}}\right]^{2}+\left[\frac{\left(\mathrm{v}-\mathrm{v}_{0}\right)_{\mathrm{r}}}{\mathrm{bC}}\right]^{2}=1}
\end{gathered}
$$

This is an equation of ellipse centered at $\left(\mathrm{u}_{0}, \mathrm{v}_{0}\right)$ rotated with an angle $\theta$ with respect to the $u$ axis. The main axis of the ellipse have length $2 a C \simeq a$ and $2 b C \simeq b$ respectively. Figure 3.10 represents the half magnitude profile in frequency domain

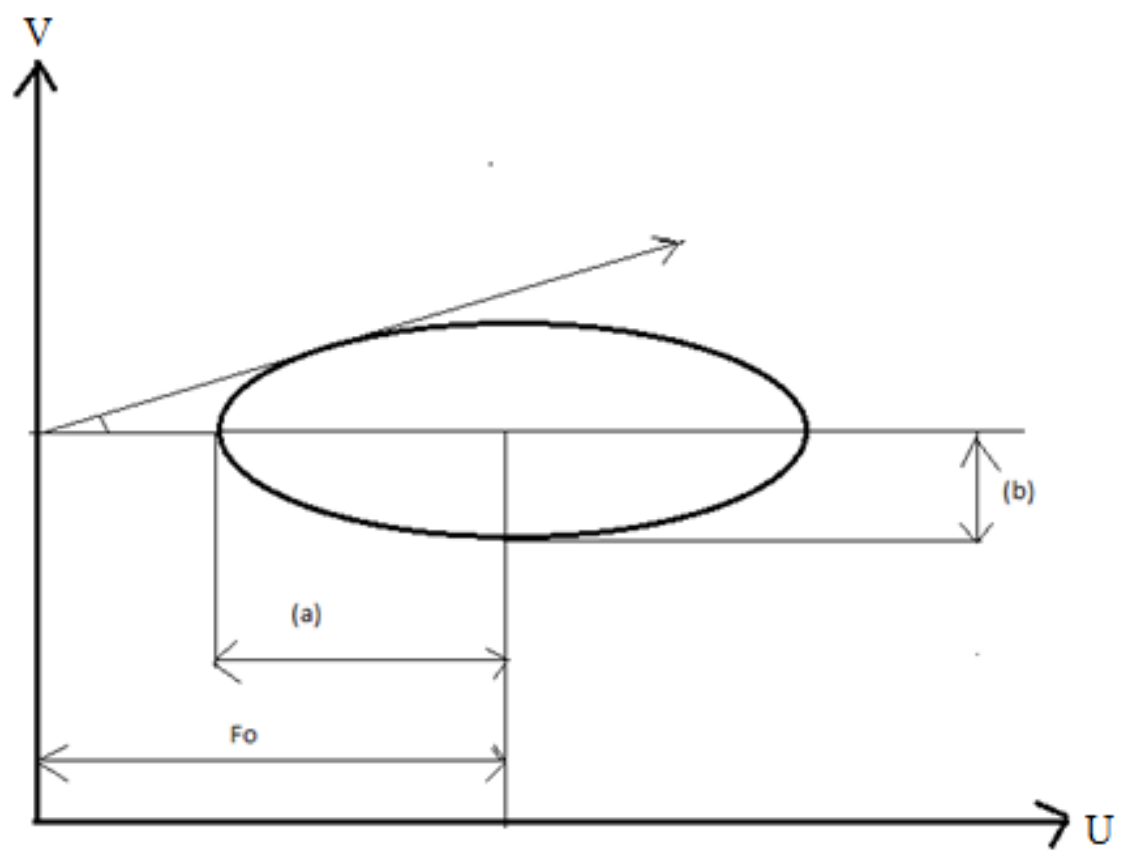

Figure 3.10: Half-Magnitude Frequency 


\subsection{Application of Gabor Filters}

Gabor filters are band pass filters which are used successful in many applications such as texture segmentation/classification, target detection, character recognition, fingerprint recognition, face recognition, image analysis and compression and edge detection. In this thesis edges in oriented structure are introduced and we utilize Gabor filter to detect them in difference orientations, hence in the following we provide the more detail of detection of edges.

\subsubsection{Edge Detection}

Edges and lines usually convey the most relevant information of an image; hence it is important to detect them in a reliable way. Edges are caused by changes in some physical properties of the surfaces being imaged, so they provide useful structural information about object boundaries, such as illumination, geometry, and reflectance. In edge detection methods, the discontinuities in the image gray level are enhanced by neighborhood operators .it has extensively analyzed in computer vision but it has not yet solved. In fact, despite considerable efforts, the ideal scheme able to detect and localize with precision edges at all scales, irrespective of their shape and configuration, has not yet been produced. Prewitt and Sobel operators use masks having the size of $3 \times 3$ pixels [26].

\subsubsection{Prewitt and Sobel Operations}

The Prewitt operators for the horizontal and vertical derivatives, $G_{x}(x, y)$ and $G_{y}(x, y)$, respectively, are defined as follows:

$$
\left[\begin{array}{ccc}
-1 & -1 & -1 \\
0 & 0 & 0 \\
1 & 1 & 1
\end{array}\right]
$$


The Sobel operators are similar to the Prewitt operators, but they include larger weights for the pixels in the row and column of the pixel being processed. The Sobel operators for the horizontal and vertical derivatives, $G_{x}(x, y)$ and $G_{y}(x, y)$, respectively, are defined as follows:

$$
\begin{gathered}
{\left[\begin{array}{rrr}
-1 & 0 & 1 \\
-2 & 0 & 2 \\
-1 & 0 & 1
\end{array}\right]} \\
{\left[\begin{array}{rrr}
-1 & -2 & -1 \\
0 & 0 & 0 \\
1 & 2 & 1
\end{array}\right]}
\end{gathered}
$$

The horizontal and vertical components of the gradient, $G_{x}(x, y)$ and $G_{y}(x, y)$, respectively, are obtained by convolving the preprocessed image with the corresponding Sobel or Prewitt operators. A commonly used definition of the combined gradient magnitude is:

$$
G(x, y)=\left[G x(x, y)^{2}+G y(x, y)^{2}\right]^{0.5}
$$

\subsubsection{The Canny Edge Detection Method}

Canny [27] proposed an approach for edge detection based upon three criteria for good edge detection, including multidirectional derivatives. Cranny's method is guided by the following requirements:

The edge detector should have a high probability of finding all edges and low probability of mistaking non-edge objects as edges, represented in the form of a signal-to-noise ratio.

The edges found by the algorithm should also be as close to the true edges as possible, represented by the root mean-squared distance of the detected edges from the true edges. 
The algorithm should have only one edge pixel when an edge is present, represented by the distance between the adjacent maxima in the output.

The Canny method implements edge detectors based upon kernel functions formed by using the first derivative of Gaussian functions. This method is computationally more complex than the Sobel operators because of its optimization procedures. 


\section{Chapter 4}

\section{GABOR FILTERS FOR BLOOD VESSELS}

\section{DETECTION IN RETINA}

\subsection{Introduction}

In this chapter, a retinal vessel extraction system that uses Gabor filter based segmentation of the vessels in retinal images, has been proposed. The proposed system also incorporates a systematic way of determining threshold value. Conceptually, several stages have been used for the retinal vessel segmentation. The main goal is to capture efficient edge information of the vessels. The system diagram of the proposed approach is shown in Figure 4.1. The proposed approach is applied to retina images from DRIVE database. The system is divided to three stages:

In first stage, each input color image was converted to 8-bit intensity scale. Then the 180 Gabor filters with the rotation separation of $1^{\circ}$ has been used to capture edge data of the vessels. Different scales are also used to capture edge information of the vessels. In order to approximately determine the vessel edge locations, maximum response of each image are selected. After Gabor filtering, image processing techniques such as logarithmic normalization is applied to suppress the impulsive values for easier post-processing.

In second stage mask processing in order to eliminate the area around of images was applied. Then morphological operation with a disk-shaped structuring element of 
radius equals to 10 pixels to thinning is applied. Then thresholding for segmentation is used. Furthermore, we have developed a systematic way to deciding efficient threshold value.

In last stage, the manually segmented image from DRIVE database is compared to segmented output. The difference between the segmented images and the manually segmented images are called the segmentation error containing error pixels. We decomposed error pixels into false positive pixels and true negative pixels corresponding to blue and red color in our illustrations. False positive and true negative pixels are used in error analysis. 


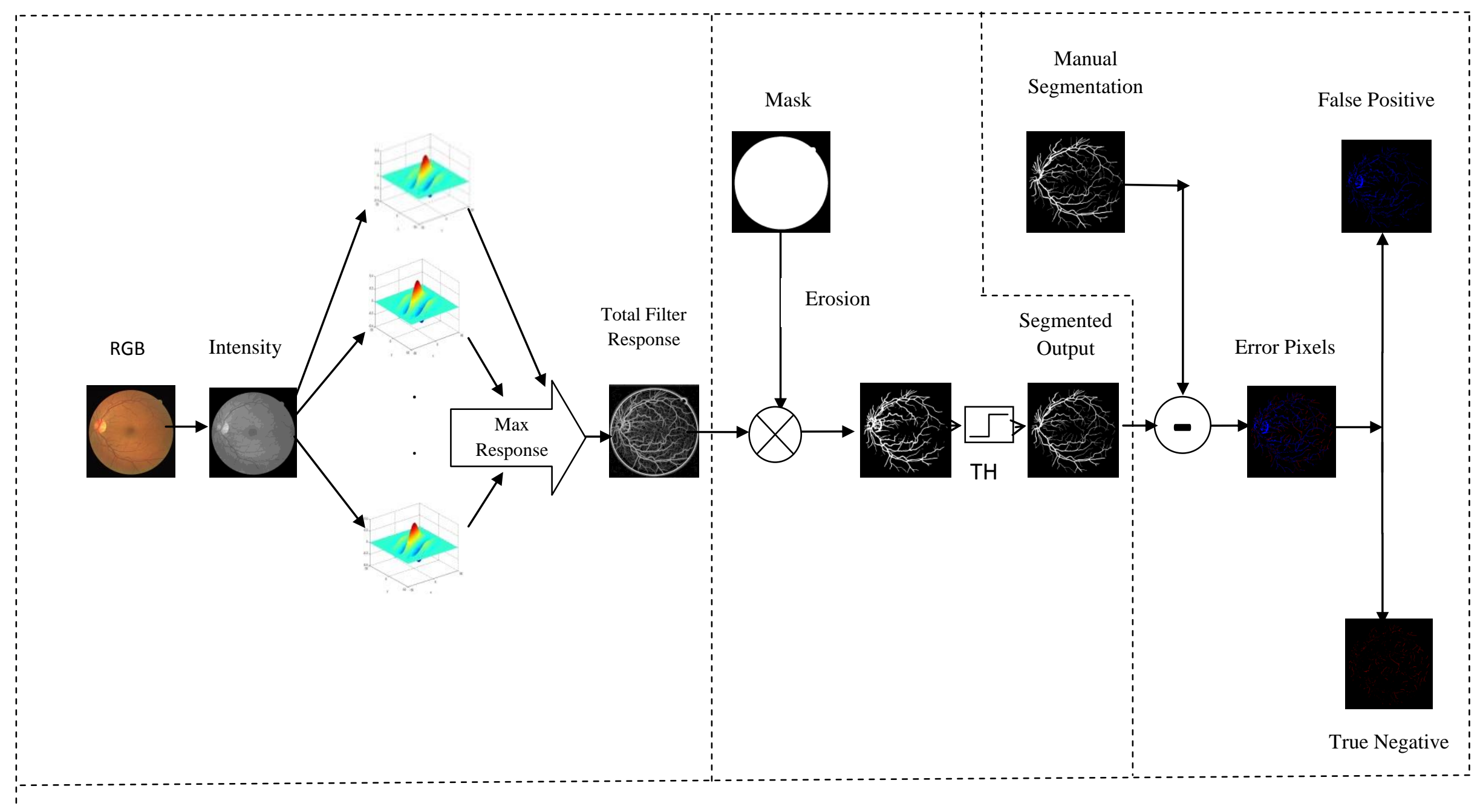

Figure 4.1: System Diagram of the Proposed Approach for Vessel Segmentation using Gabor Filter 


\subsection{Experimental Methodology}

In order to evaluate segmentation performance and to get an understanding of its use, this part of the thesis introduces some of the notations surrounding the segmentation process. In general, four possible decisions and two types of errors are made when comparing the automatic segmentation result with the manual segmentation as shown in Table 4.1.

Table 4.1: Relations between the Measurement Probabilities of the Manual Segmentation and Automatic Segmentation [34].

\begin{tabular}{|c|c|c|c|}
\hline \multirow{2}{*}{ Manual } & \multicolumn{3}{|c|}{ Automatic Segmentation } \\
\cline { 2 - 4 } & Positive & Negative & Total \\
\hline Positive & True Positive (TP) & False Positive (FP) & TP+FP \\
\hline Negative & False Negative (FN) & True Negative (TN) & FN+TN \\
\hline Total & TP+FN & FP+TN & TP+FN+FP+TN \\
\hline
\end{tabular}

TP: If both manual segmentation (Ground truth) and automatic segmentation are positive.

FN: If the manual segmentation (Ground truth) is positive and the automatic segmentation is negative.

FP: If the manual segmentation (Ground truth) is negative and the automatic segmentation is positive.

TN: If both manual segmentation (Ground truth) and automatic segmentation are negative.

In Figure 4.2, given that the result of the proposed segmentation is represented by set $\mathrm{A}$, and the result of manual segmentation (Ground Truth) is represented by $\mathrm{B}$, the 
following relationships can be generated. Note that the mask refers to the total set where both $\mathrm{A}$ and $\mathrm{B}$ are bounded.
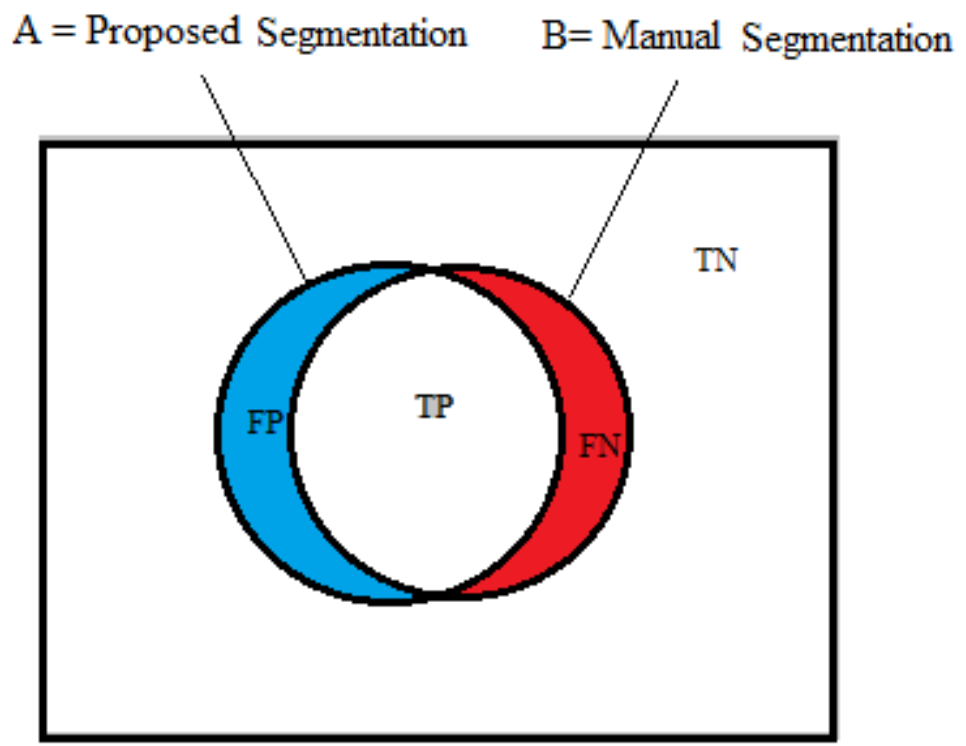

Figure 4.2: Illustration of the Measure of Overlap between Two Segmentations where A Is Proposed Segmentation and B Is Manual Segmentation

$$
\begin{gathered}
\mathrm{TP}=\mathrm{A} \cap \mathrm{B} \\
\mathrm{FP}=(\mathrm{A} \cup \mathrm{B})-\mathrm{B} \\
\mathrm{FN}=(\mathrm{A} \cup \mathrm{B})-\mathrm{A} \\
\mathrm{TN}=\mathrm{Mask}-(\mathrm{A} \cup \mathrm{B}) \\
\mathrm{TPR}=\frac{\mathrm{TP}}{(\mathrm{TP}+\mathrm{FN})} \\
\mathrm{FPR}=\frac{\mathrm{FP}}{(\mathrm{FP}+\mathrm{FN})} \\
\mathrm{FNR}=\frac{\mathrm{FN}}{(\mathrm{FN}+\mathrm{PR})} \\
\mathrm{TNR}=\frac{\mathrm{TN}}{(\mathrm{TN}+\mathrm{FP})}
\end{gathered}
$$




\subsubsection{Sensitivity and Specificity}

The sensitivity and specificity may provide a better assessment of the overall performance of a result. The sensitivity of detection is calculated as the ratio of the number of images with successful detection of the blood vessels to the number of images segmented.

$$
S E=\frac{T P}{(T P+F N)} \times 100
$$

Sensitivity measures the proportion of correctly identified ground truth, and specificity measures the proportion of correctly automatic segmentation result.

$$
S P=\frac{T N}{(T N+F P)} \times 100
$$

Sensitivity is equivalent to the true-positive ratio (TPR), and specificity is equivalent to the true-negative ratio (TNR).

\subsubsection{Segmentation Accuracy}

Accuracy is a measure of the proportion of decision performance including the concepts of the "sensitivity" and "specificity" of a diagnostic test. In fact, sensitivity and specificity represent two kinds of accuracies. These measures and the related indices, TPR and FPR, are more meaningful than "'accuracy", yet do not provide a unique description of diagnostic performance because they depend on the arbitrary choice of a decision threshold.

$$
\text { accuracy }=\frac{\mathrm{TP}+\mathrm{TN}}{(\mathrm{TP}+\mathrm{TN}+\mathrm{FP}+\mathrm{FN})}
$$

\subsubsection{ROC Analysis}

Receiver operating characteristic (ROC) curve analysis allows visual evaluation of the trade-offs between sensitivity and specificity associated with different values of 
the test result. Also it can be used to select the optimal threshold for a particular application. A ROC curve is plotted on a 2-dimensional unit square plot $\mathrm{y}$-axis and $\mathrm{x}$ axis correspond to the TPR (or sensitivity) and correspond to the FPR (1 specificity) are, respectively and close to the point $(0,1)$. The area under a ROC curve (AUC) also is called $\left(A_{z}\right)[29]$.

In Figure 4.3 the solid line represents the relationship between the sensitivity and specificity as the discrimination threshold of the test result is varied. This can be compared with the line of no-discrimination (dash line), and the red line depicting an ideal result.

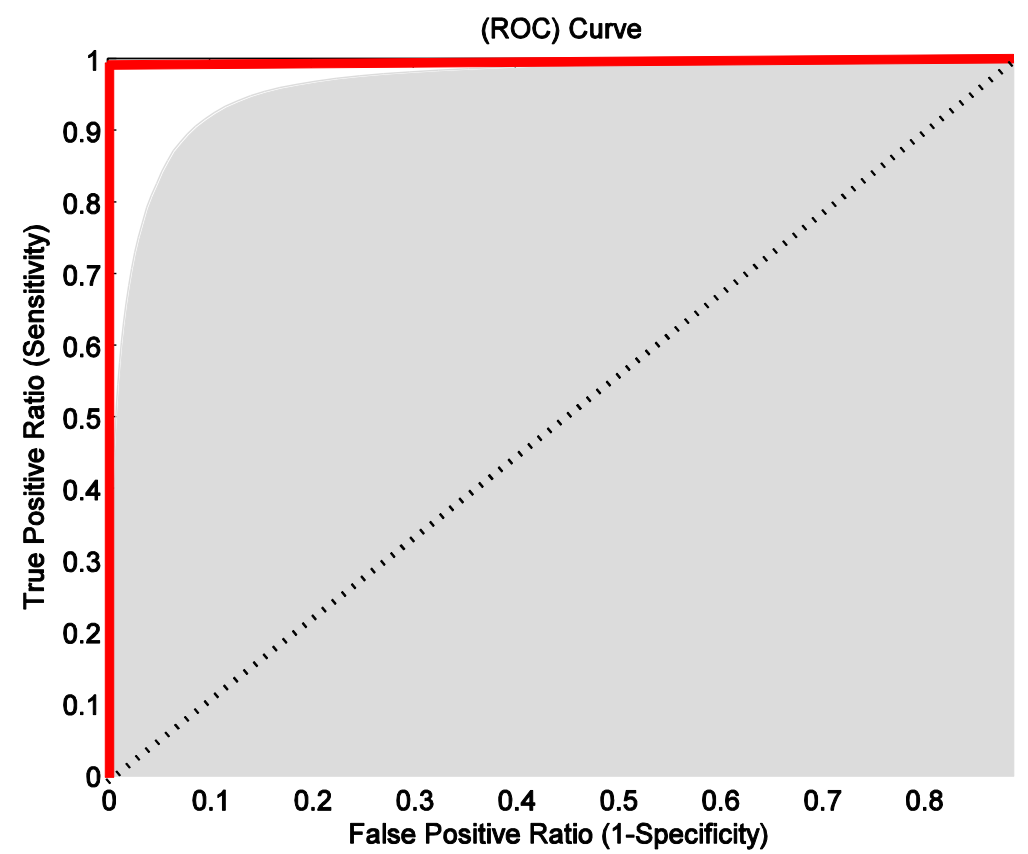

Figure 4.3: Illustration of the ROC Curve for a Binary Result.

\subsubsection{Thresholding (False Acceptance / False Rejection)}

The False Acceptance Rate (FAR) or False Alarm Rate usually refers to the expectancy of the False Positive Ratio (FPR). The False Rejection Rate (FRR) refers to the expectancy of the False Negative Ratio (FNR). 


\subsubsection{Equal Error Rate (EER)}

The first objective approach which is a biometric security system that determines the threshold values for its FAR and FRR, if the score distributions overlap the FAR and FRR intersect at a certain point. The value of them at this point, is named the Equal Error Rate (EER). In Figure 4.4 horizontal and vertical axis represent value of threshold and corresponding Equal Error Rate, respectively. The value of this cross point is corresponding to a threshold which we choose as the optimal threshold.

The EER of a system can be used to give an optimal threshold. We proposed a systematic way of determining of threshold value which can develop system performance.

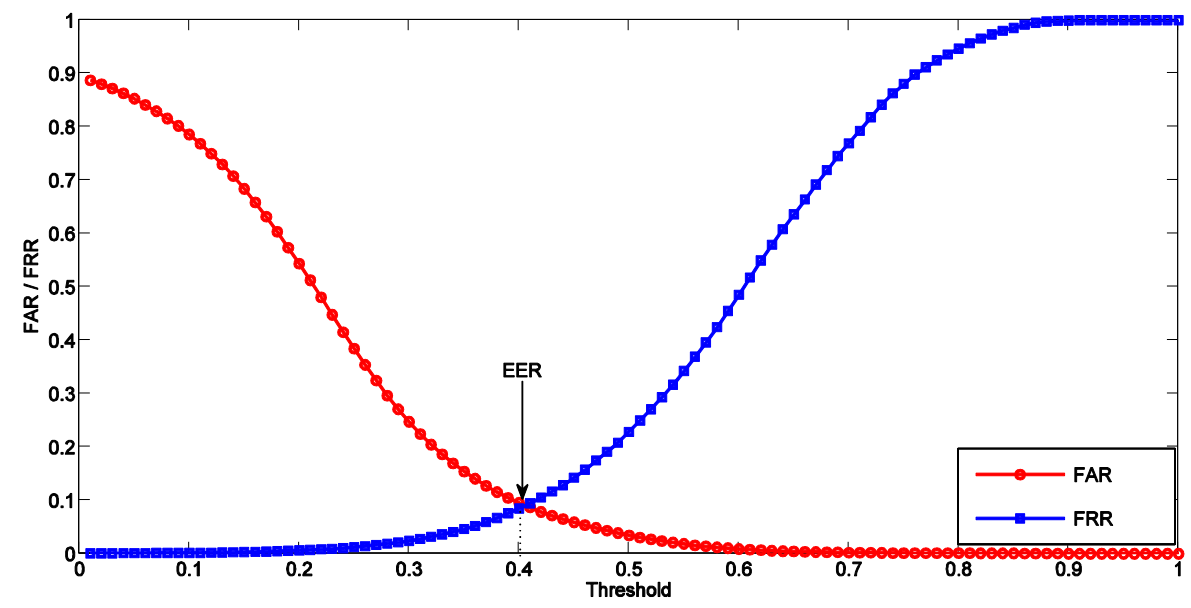

Figure 4.4: Equal Error Rate (EER)

\subsubsection{Total Error Rate (TER)}

The lower the EER is the better performance of the system, as Total Error Rate (TER) which is the sum of the FAR and the FRR at the point of the EER decreases. 
In fact TER is our second approach which has been used extensively for comparison of biometric verification performances. Figure 4.5 illustrates False Acceptance Rate (FAR) and False Rejection Rate (FRR) and the Total Error Rate (TER). The threshold minimizing the TER is an alternative approach to determine a suitable threshold. In addition to the threshold determined by EER, the threshold corresponding TER can be used as an efficient threshold level for segmentation.

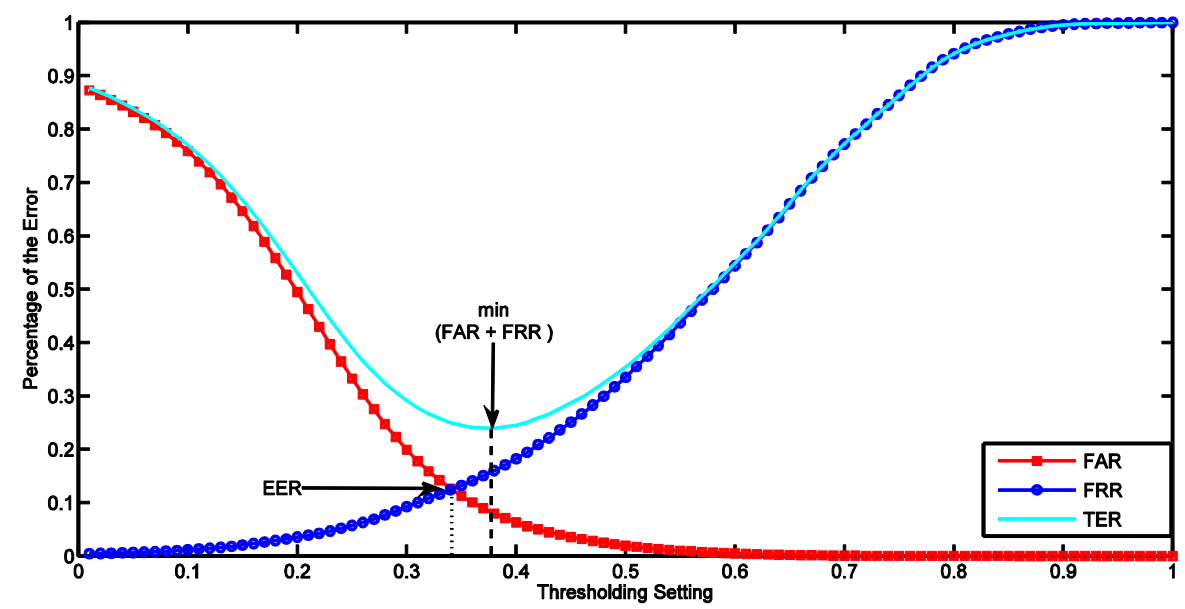

Figure 4.5: Biometric Systems Are Rated on the Basis Performance Standards

\subsection{Retina Database}

At the best of our knowledge, two publicly available data sets with retinal images (with and without diseases) can be found: STARE [37] and DRIVE [9] databases. Due to the content and type of images in these databases, their use is subject to the kind of application under study.

The DRIVE database (Digital Retinal Images for Vessel Extraction data base) contains 40 available images with and without diseases, which allows a more general analysis of the applications, algorithms or methodologies under study. The main goal 
of the DRIVE database is to establish and facilitate comparative studies to segmentation of retinal blood vessels detection in medical images. In this thesis, the DRIVE database has been selected for detection retina blood vessels which has represented in Figure 4.6. (a) is the original image number one of the test set and (b) is the mask. Also (c) and (d) are manual segmentation of the first and second observe, respectively. The images were acquired using a Canon CR non-mydriatic camera with three charge-coupled device (CCD) detectors with a 45 degree field of view (FOV).

The dataset consists of a total of 40 color fundus photographs. Each image is of size 584 by 565 pixels, represented using 24 bits per pixel in the standard RGB format. Considering the size and FOV of the images, they are low-resolution fundus images of the retina, having an approximate spatial resolution $20 \mu \mathrm{m}$ per pixel [28]. 


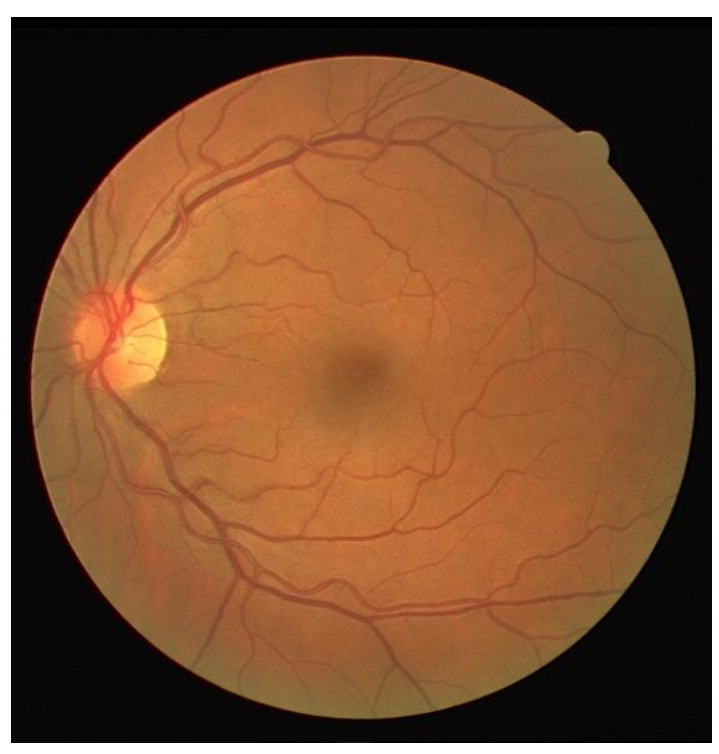

(a)

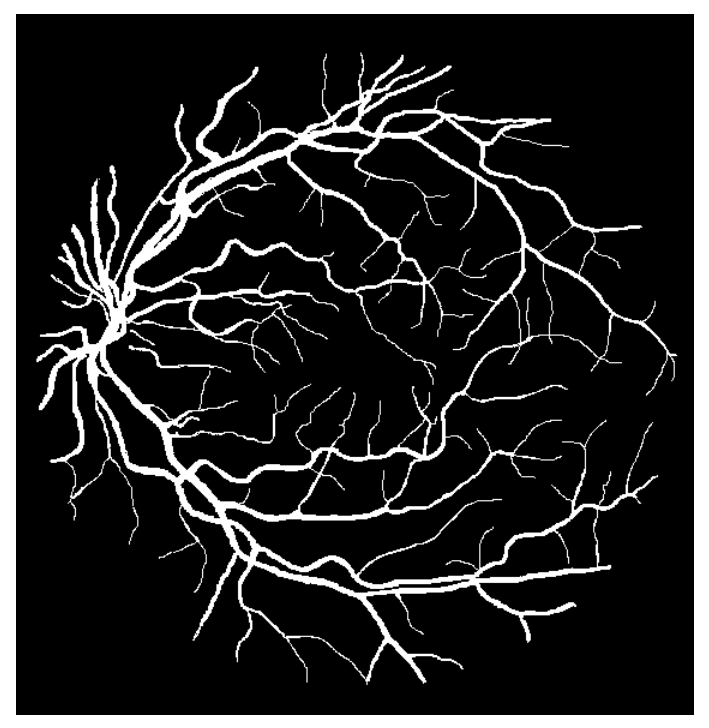

(c)

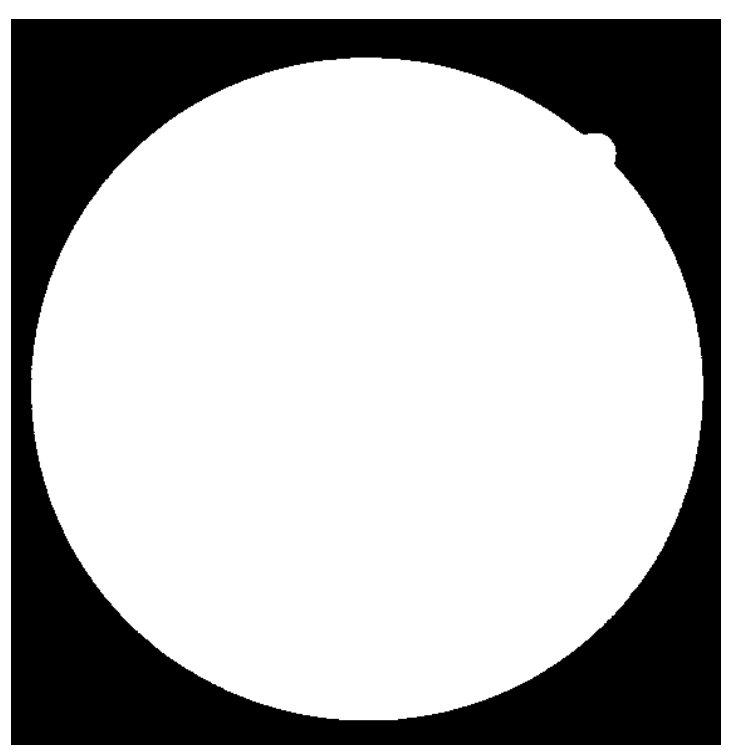

(b)

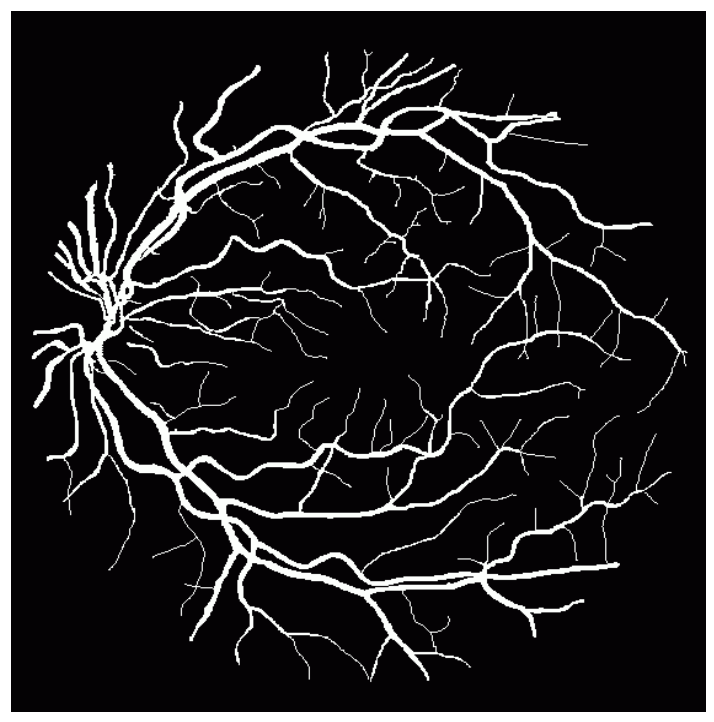

(d)

Figure 4.6: (a) Image 01 of the Test Sets. (b) The Mask Delimiting the FOV. (c) The Manual Segmentation of the First. (d) The Second Observer [9] 


\subsection{Proposed Method}

\subsubsection{Gabor Filter Based Vessel Detection}

In this thesis we propose Gabor filter for detection of blood vessels. In first stage different image processing methods have used for capturing data from vessels. Methods include edge detection and logarithm normalization. The detection process can be carried out either on the original images from DRIVE database.

\subsubsection{Pre-processing}

The images in the DRIVE datasets are provided in the $[R, G, B]$ format, where $R$, $\mathrm{G}$, and B correspond the red, green, and blue components, respectively, of the color image. In the color retinal images, blood vessels appear darker than the background. So, it is essential to exempt the vessel area during the detection of lesions to avoid false positives. The Figure 4.7 shows that the blood vessels appear most contrasted in the green channel compared to red and blue channels in RGB image, so the green channel image has been used for further processing suppressing the other two color components. In this stage, for intensity normalization we have divided each pixel of the original color image by 255 . 


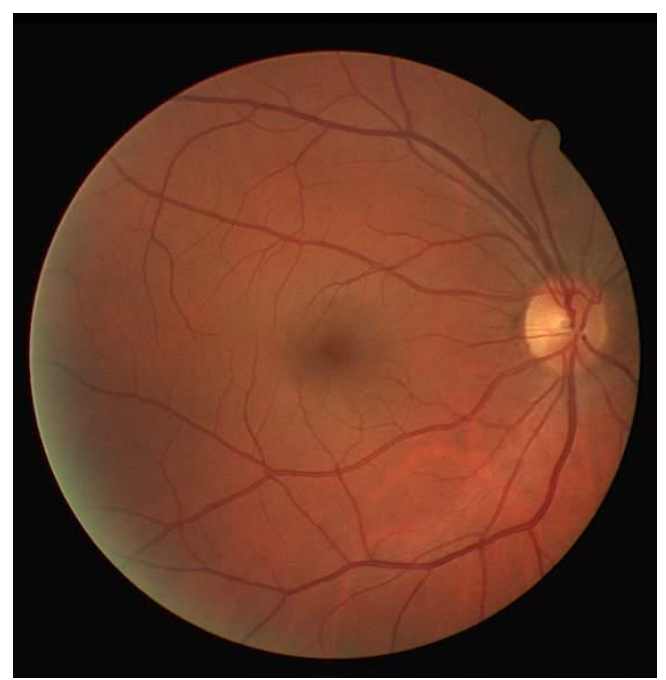

(a)

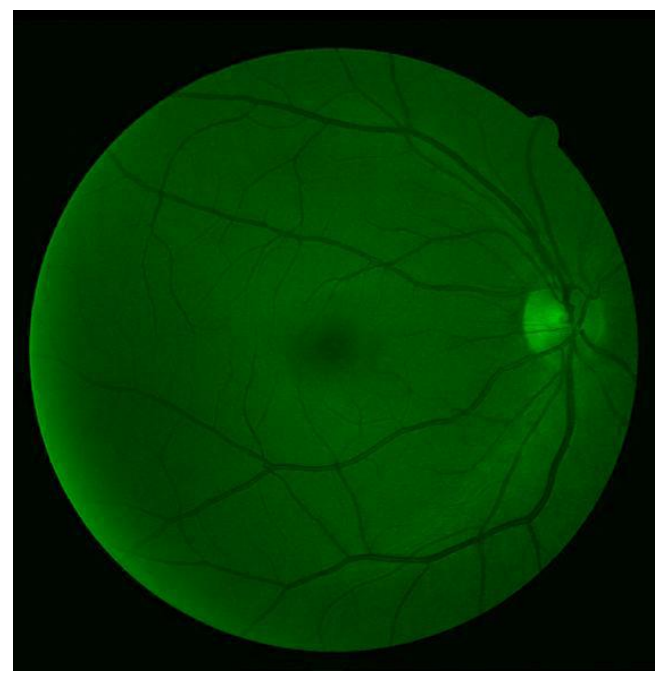

(c)

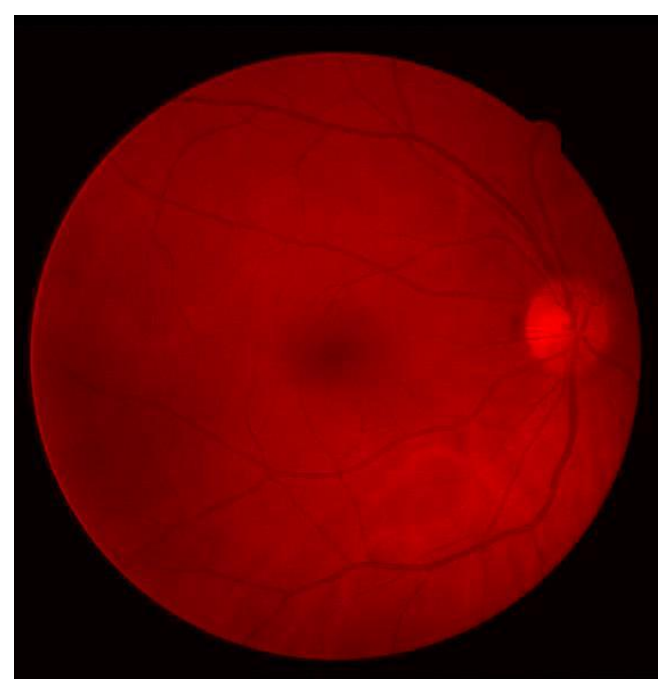

(b)

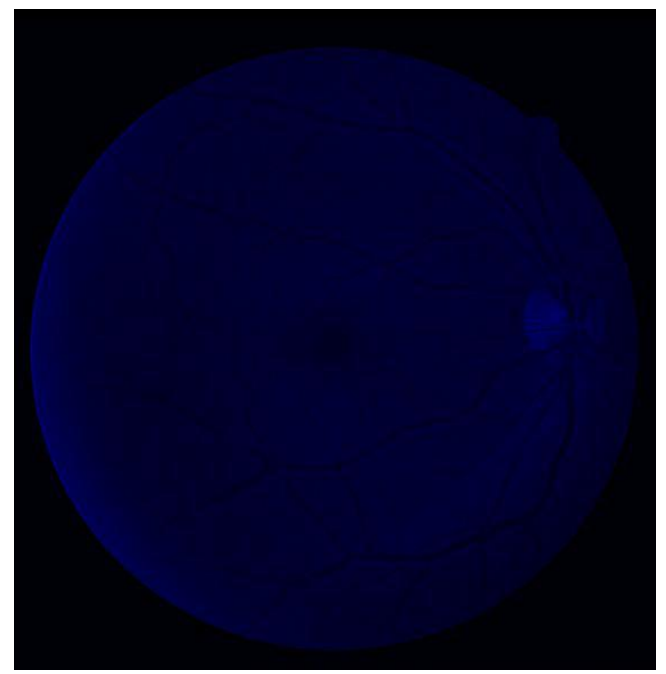

(d)

Figure 4.7: Appearance of Vessels in Different Color Channels. (a) Color Retinal Image. (b) Red Channel Image. (c) Green Channel Image. (d) Blue Channel Image[10]

\subsubsection{Gabor Filter for Detecting Oriented Patterns}

The Gabor filters are widely applied to image processing and computer vision application problems such as texture segmentation and face recognition. Since, the vessels in the retinal image are connected and piecewise linear, for their segmentation Gabor filters are better suited as they are capable of detecting oriented features and can be fine tuned to specific frequencies. Gabor functions have been found to provide good models for the receptive fields of simple cells in the striate 
cortex. Gabor functions provide optimal joint resolution in both the Fourier and time space, and form a complete basis set through phase shift and scaling or dilation of the original mother wavelet function.

We have applied the Gabor filtering techniques to detect blood vessels in retina images based upon Gabor wavelets [31]. The real Gabor filter kernel (or mother wavelet) oriented at the angle $\theta=\pi$ can be formulated as [39]:

$$
g(x, y)=\frac{1}{2 \pi \sigma_{x} \sigma_{y}} \mathrm{e}^{\left(-\frac{1}{2}\left(\frac{x^{2}}{\sigma_{\mathrm{x}}^{2}}+\frac{y^{2}}{\sigma_{\mathrm{y}}^{2}}\right)\right)} \cos \left(2 \pi f_{0} \mathrm{x}\right)
$$

According this formula $\sigma_{x}$ and $\sigma_{y}$ are the standard deviation values in the $x$ and $y$ directions, and $f o$ is the frequency of the modulating sinusoid. Kernels at other angles are obtained by rotating the mother wavelet. In this thesis, we use of 180 Kernels with angles spaced evenly over the range $[-\pi / 2, \pi / 2]$ by using the coordinate transformation

$$
\begin{aligned}
x^{\prime} & =x \cos \theta+y \sin \theta \\
y^{\prime} & =-x \sin \theta+y \cos \theta
\end{aligned}
$$

Where $\left(x^{\prime}, y^{\prime}\right)$ is the set of coordinates rotated by the angle $\theta$. The parameters in equation above, namely $\sigma_{x}$ and $\sigma_{y}$ and $f o$, need to be derived by taking into account the size of the lines or curvilinear structures to be detected. $\tau$ is the thickness of the line detector. These parameters constrain $\sigma_{x}$ and $f_{o}$ are as follows:

The amplitude of the Gaussian term in equation (4.12) is reduced to one half of its maximum at $x=\frac{\tau}{2}$ and $y=0$; therefore, $\sigma_{x}=\tau /(2 \sqrt{2} \ln 2)=\tau / 2.35$. 
The cosine term has a period of $\tau$; hence $f_{o}=\frac{1}{\tau}, \sigma_{y}=1 / \tau$. The value of $\sigma y$ could be defined as $\sigma_{y}=l \sigma_{x}$, where $l$ determines the elongation of the Gabor filter in the orientation direction, with respect to its thickness is varied to prepare a bank of filter at different scales for multiresolution filtering and analysis value of $\tau$ could be varied to prepare a bank of filters at different scales for multiresolution filtering and analysis [31].

\subsubsection{Maximum Response and Logarithm Normalization}

To obtain good response of different directions, the Gabor filter is rotated from $0^{\circ}$ to $180^{\circ}$ in the steps of one degree to produce a single peak response on the center of a vessel segment. At each pixel only the maximum response is retained. Then logarithmic normalization on the maximum response is applied to suppress the peak value of filter response. 


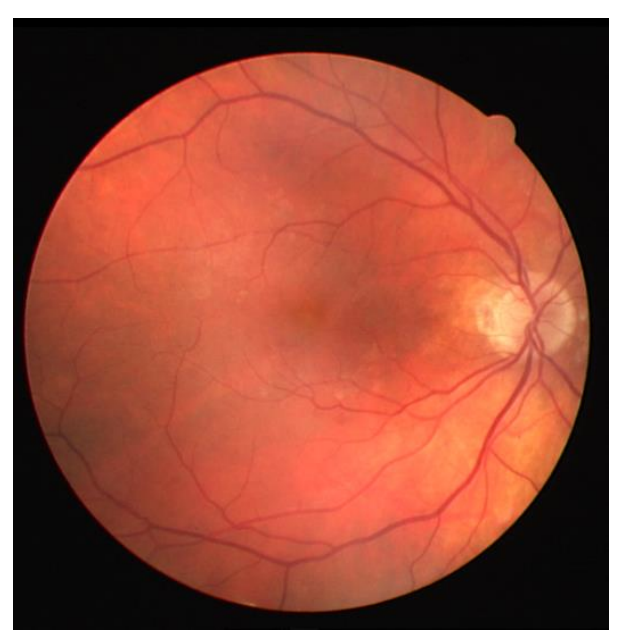

(a)

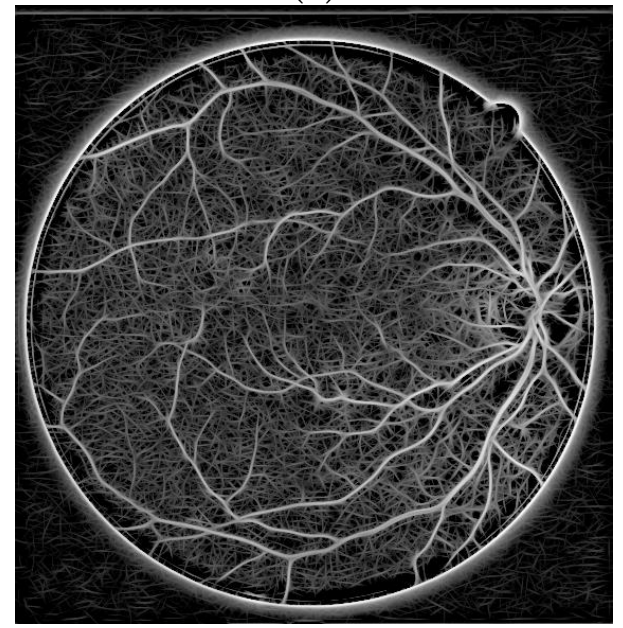

(c)

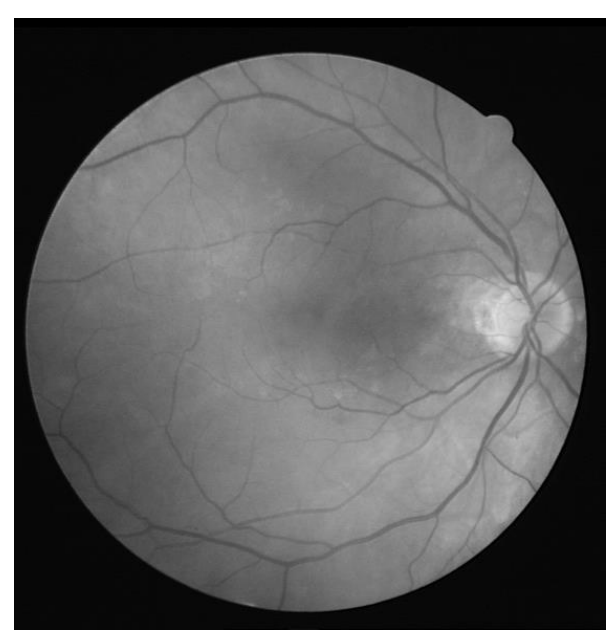

(b)

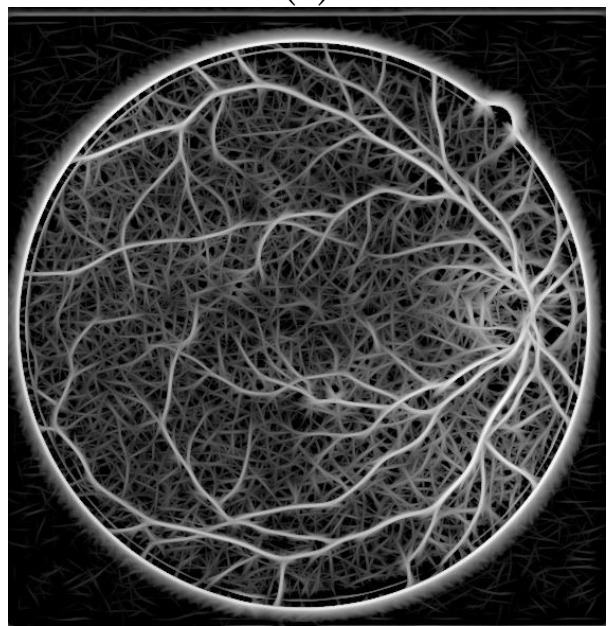

(d)

Figure 4.8: (a) Image 07 Test Set (b) Input Intensity Image. Magnitude Response of 180 Gabor Filters over $0^{\circ}-180^{\circ}$ before Thresholding. (c) $\tau=4$ Pixels, $1=2.5$. (d) $\tau=$ 8 Pixels, $1=2.5$

Figure 4.8 (a) shows an image (number 07) from the DRIVE database [9]. (b) is intensity component of the original color image. The result in (c) was obtained using a bank of 180 Gabor filters without threshoding with $\tau=4$ pixels and $l=2.5$; that in (c) was obtained with $\tau=8$ pixels. The magnitude response image was composed by selecting the maximum response over all of the Gabor filters for each pixel. The result in Figure 4.8 (c) indicates that the filters have detected only the edges of the thick vessels, with poor response along their center-lines. On the contrary, the result in Figure 4.8 (d) shows that the thick vessels have been detected well; some of the thinner vessels have not been detected. The results indicate the 
need for multiscale or multiresolution filtering and analysis, which is easily facilitated by the proposed design of the Gabor wavelet.

\subsubsection{Segmentation by Thresholding}

Thresholding allows the separation of an image into separate components by turning it into a binary image. This involves the image being separated into white or black pixels on the basis of whether their intensity value is greater or less than a certain threshold level. The process of thresholding maybe particularly useful to remove unnecessary detail or variations and highlight detail that is of interest.

\subsubsection{Thresholding Operation}

In second stage to classify the pixels into vessels and non vessels thresholding is applied. The effective region of the image was detected using the normalized threshold of 0.43 . The calculation of the thresholds will be explained in a systematic manner in the proceeding stage.

\subsubsection{Morphological Erosion Operation}

Morphological operation [26] include the basic operations of erosion and dilation, and modifications and combinations of these operations. Basically, all morphological filters are neighborhood-based operators with a structuring element for the neighborhood. In this thesis erosion has applied to binary images are restricted to 0 or 1. Erosion removes pixels from an image, or, equivalently, turns pixels that were originally 1 to 0 . Erosion of image or set A by a structuring element $\mathrm{B}$ is denoted by $\ominus$. Erosion for binary images may be expressed by: 


$$
\mathrm{A} \ominus \mathrm{B}=\{\mathrm{y} \mid(\mathrm{B}) \mathrm{y} \subseteq \mathrm{A}\}
$$

The operation equals the set of elements y for which all translations of $\mathrm{B}$ by $\mathrm{y}$, denoted by $(B)_{y}$, are inside A.

\subsubsection{Mask Processing}

In this part, the artifacts present at the edges of the masks generated for images from the DRIVE dataset were removed by applying morphological erosion with a discshaped structuring element of diameter 10 pixels. Figure 4.9 indicates overview of the main steps taken by our algorithm when processing a retinal image. 


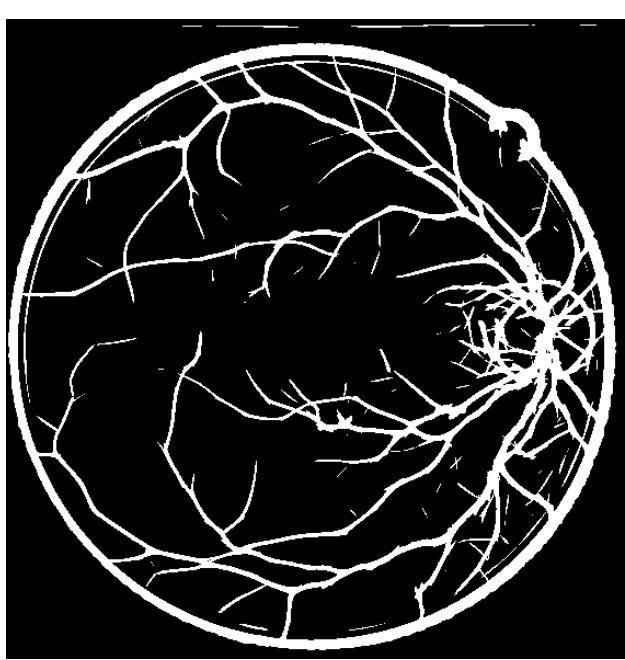

(a)

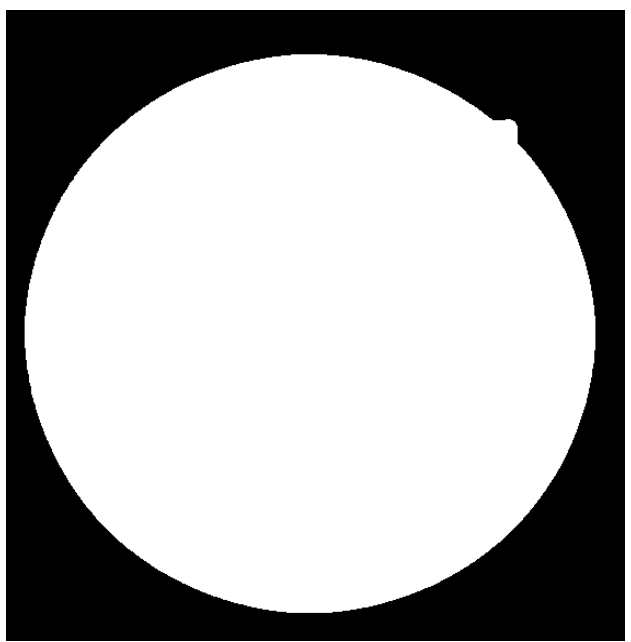

(c)

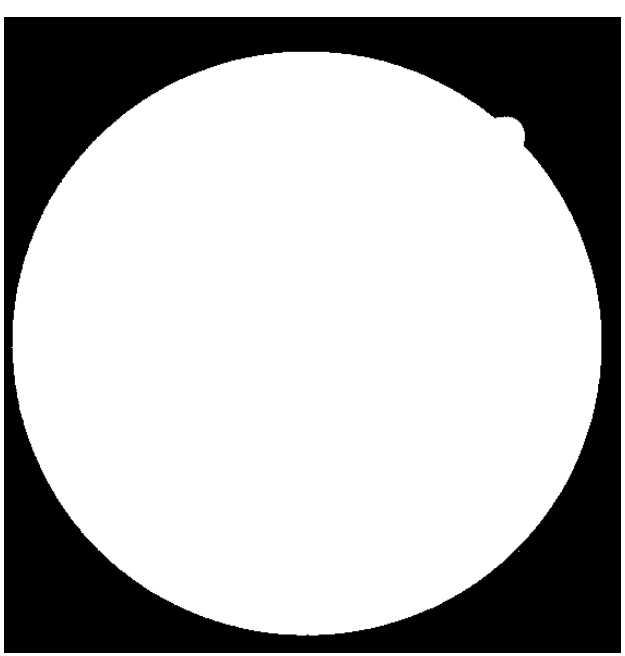

(b)

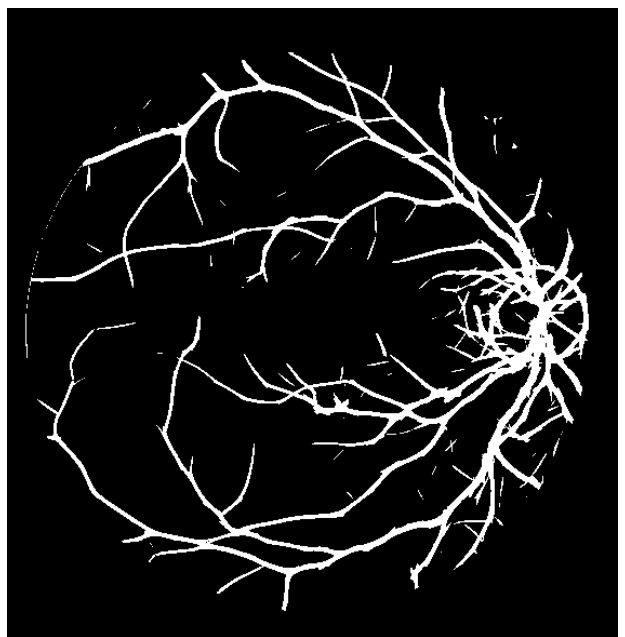

(d)

Figure 4.9: (a) The Image 07 Normalized by Threshold of 0.43. (b) The Mask Image 07 (c) Morphological Thinning Is Applied to (b). (d) The Area Around of Image Is Eliminated with Mask

\subsubsection{Error Analysis}

In final stage we analyze error by comparing segmented image (from pervious stage) with manual segmentation (ground truth). The result achieved is called Error image analysis. Indeed, image achieved is difference between segmented image and ground truth. For analyzing Error image has been divided to images. Images concluding red and blue pixels are corresponding to False Positive error pixels and False Negative 
error pixels. On the other hand if the vessels classified be in ground truth represent red pixels in Error image and if the vessels classified not be in ground truth represent blue pixels in Error image. The performance of this approach is evaluated on DRIVE databases with manually segmentation (ground truth).

The overview of the main third stage taken by our algorithm when processing a retinal image is shown in Figure 4.10.

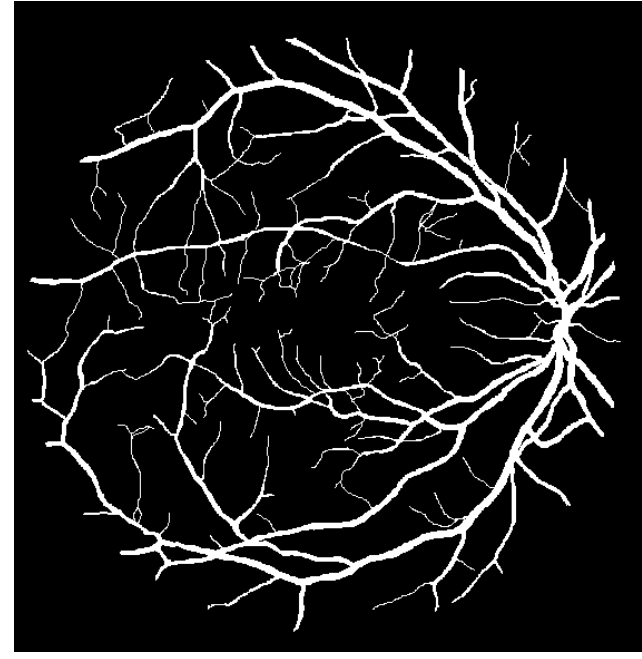

(a)

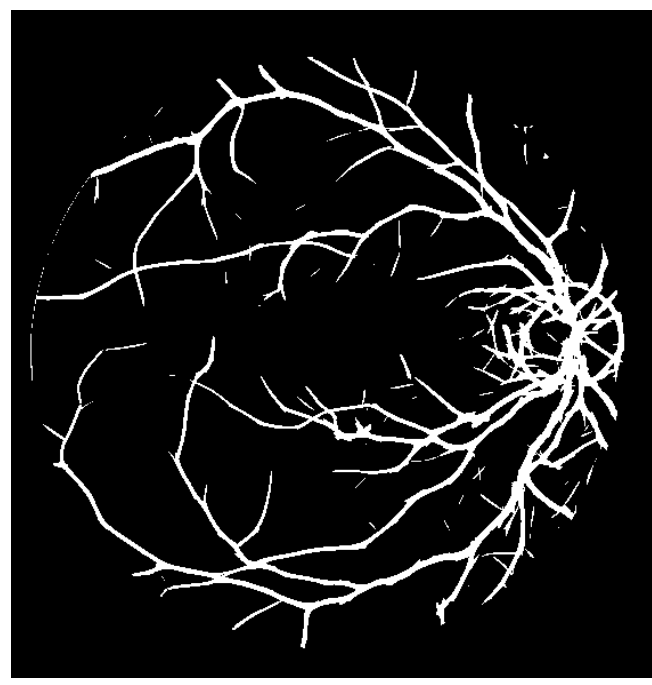

(c)

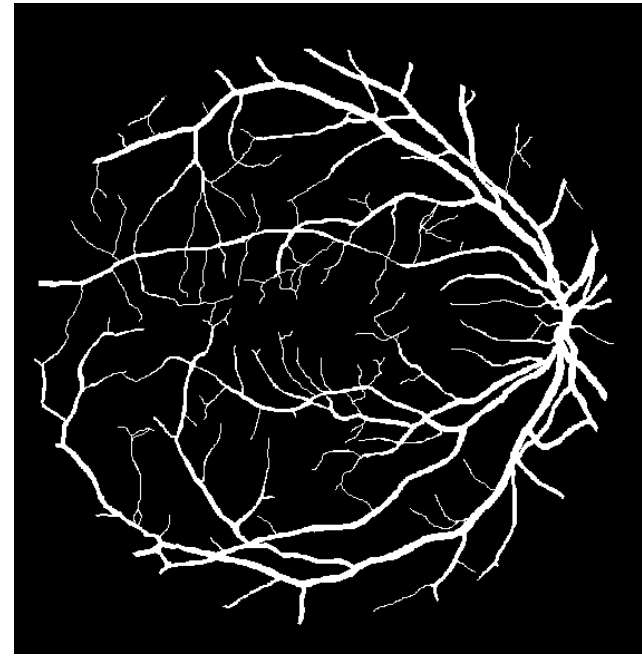

(b)

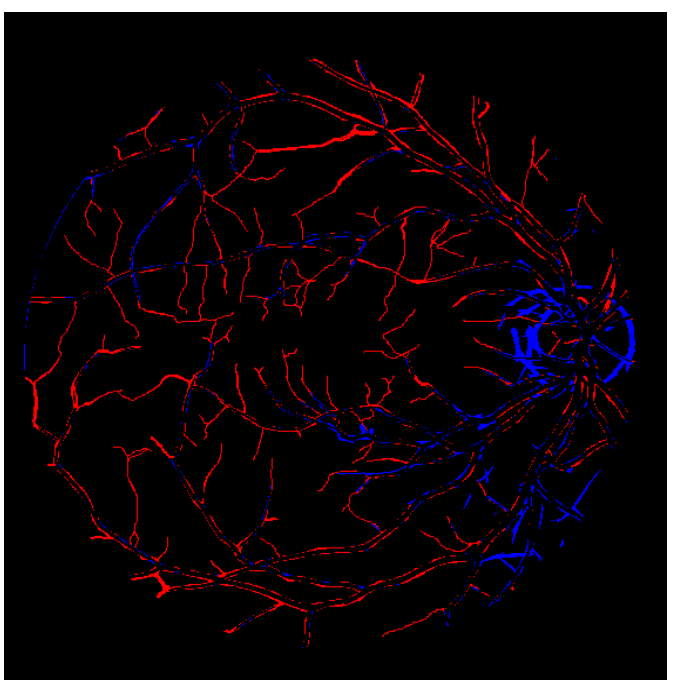

(d) 


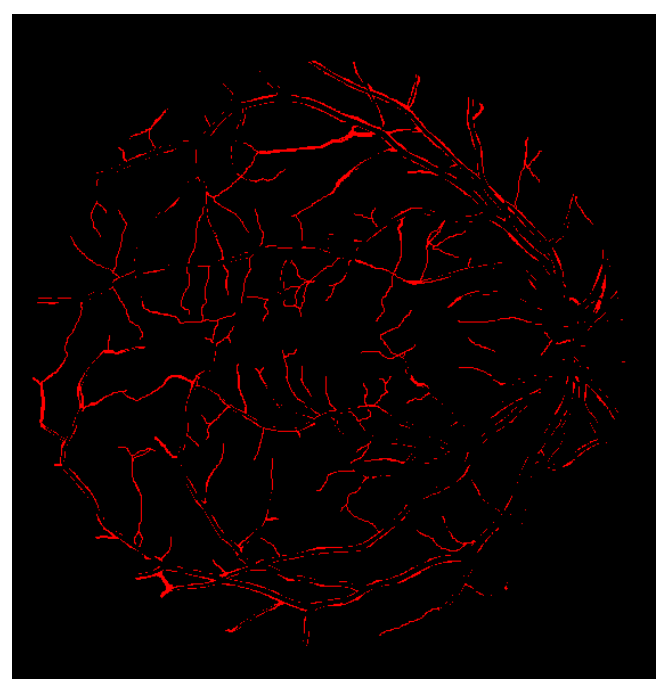

(e)

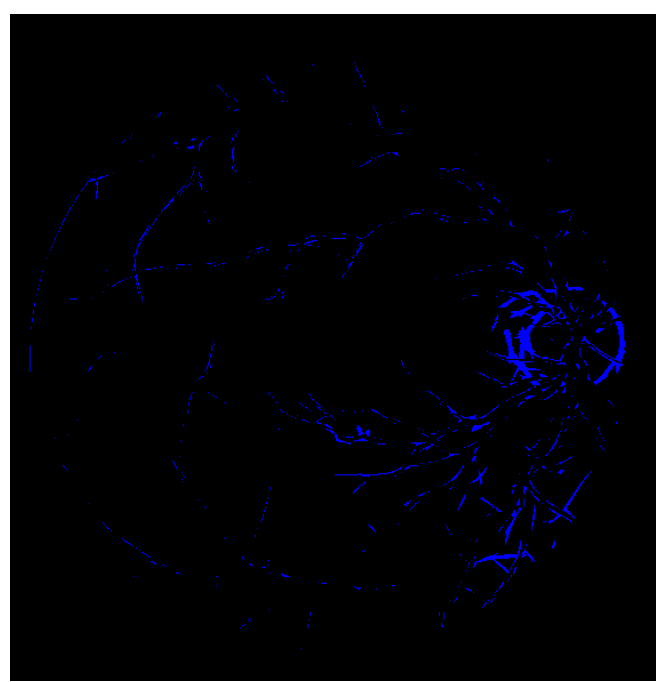

(f)

Figure 4.10: (a) The Manual Image 07 Test Set. (b) Morphological Thinning Applied at the Boundaries (a). (c) The Segmented Output Image. (d) Comparison between Image (b) and Image (c) Containing Error Pixels. (e) The Red Pixels Corresponding to False Positives. (f) The Blue Pixels Corresponding to False Negatives.

\subsubsection{Mathematical Description of the Proposed Algorithms:}

- $\quad$ Let $\Gamma(x, y)$ to be the input retina image.

- $I(x, y)$ which is the intensity image is calculated by

$$
I(x, y)=\frac{R_{\Gamma(x, y)}+G_{\Gamma(x, y)}+B_{\Gamma(x, y)}}{3}
$$

Where $\mathrm{R}_{\Gamma(x, y)}, \mathrm{G}_{\Gamma(x, y)}$ and $\mathrm{B}_{\Gamma(x, y)}$ correspond the red, green and blue components of the input RGB image.

- The Gabor filter $g_{\theta}(x, y)$ is applied to image $I(x, y)$ for 180 different angles $\theta$ between $-\frac{\pi}{2}$ and $\frac{\pi}{2}$ and a set of filter responses are collected by:

$$
G_{\theta}(x, y)=I(x, y) * g_{\theta}(x, y)
$$


- Where $*$ corresponds to the convolution operation. The response for each pixel position $(x, y)$ is determined by taking the maximum response among all of the 180 filters at the respected pixel position.

$$
R(x, y)=\max \left[G_{\theta}(x, y)\right], \theta=\left[-\frac{\pi}{2}: \frac{\pi}{180}: \frac{\pi}{2}\right]
$$

- Where $\theta$ is varied by increments of $\frac{\pi}{180}$ between $-\frac{\pi}{2}$ and $\frac{\pi}{2}$. Apply logarithmic normalization to suppress the peak values.

$$
\hat{R}(x, y)=\ln (R(x, y))
$$

- Use Mask to isolate retina region to segment $\Omega(x, y)$ by multiplying (pixel by pixel) with the $\hat{R}(x, y)$ with the binary mask after erosion.

$$
\Omega(x, y)=\hat{R}(x, y)[M(x, y) \ominus B]
$$

- Where $M(x, y)$ is the binary mask and $\ominus$ is the morphological erosion operation by using the circular structuring element $\mathrm{B}$ of radius 10 pixels. The values of $\Omega(x, y)$ can be thresholded to generate the binary output image $\Phi(\mathrm{x}, \mathrm{y})$.

$$
\Phi(\mathrm{x}, \mathrm{y})= \begin{cases}1 & \text { if } \Omega(x, y) \geq \psi_{t h} \\ 0 & \text { if } \Omega(x, y)<\psi_{t h}\end{cases}
$$

- Where $\psi_{t h}$ is the threshold value used. The generated output vessel image $\Phi(\mathrm{x}, \mathrm{y})$ is compared with the manually segmented vessel image $\Phi_{\mathrm{M}}(\mathrm{x}, \mathrm{y})$ for error analysis :

$$
E(x, y)=\Phi(\mathrm{x}, \mathrm{y})-\Phi_{\mathrm{M}}(\mathrm{x}, \mathrm{y})
$$




$$
\begin{aligned}
& \varepsilon_{F A}=E(x, y) \text { if } E(x, y)>0 \\
& \varepsilon_{F R}=E(x, y) \text { if } E(x, y)<0
\end{aligned}
$$

Where $\varepsilon_{F A}$ corresponds the False Alarm Error (FAR) pixels and $\varepsilon_{F R}$ corresponds the False Rejection Error (FRR) pixels. 


\section{Chapter 5}

\section{SIMULATIONS, RESULTS AND DISCUSSIONS}

\subsection{Introduction}

In this chapter according to experimental methodology we propose three methods for calculating False Positive Ratio (FPR), True Positive Ratio (TPR), accuracy, specificity (SP) and Area Under the Curve (AUC). Then we compare these approaches and results using Tables and Figures. Furthermore we compare our method with alternative methods using blood vessel detection and segmentation algorithm tested using DRIVE database [9].

\subsection{Proposed Threshold Determination}

Approach 1: In this approach firstly, all possible threshold values are studied by calculating the error between the automatically segmented blood vessel and manually segmented blood vessel. The errors correspond to the false alarm errors and false rejection errors. Then the cross point of False Acceptance Rate (FAR) and False Rejection Rate (FRR), which is referred to as the Equal Error Rate (EER) is obtained. Finally according to the obtained EER, we can determine the threshold for this approach and calculate all the relevant analysis parameters.

Approach 2: In this approach an alternative threshold is estimated. The method again uses the false alarm errors and false rejection errors in the threshold estimation process. A new curve is formed by using the sum of the FAR and FRR rates. The 
generated rate is plotted for every possible threshold value. This curve corresponds to the total error per each possible threshold value. The minimum in this curve corresponds to the minimum total error and it is named as the Total Error Rate (TER). The minimum threshold value on this curve generated the maximum performance on the segmentation performance.

Approach 3: In the last approach we have utilized a threshold according to the threshold value that maximizes the accuracy metric. Accuracy which is defined in the previous chapter is plotted against every possible threshold value and the threshold maximizing this curve is declared to the selected threshold.

Extensive studies have been done using all these three approaches for performance comparison. 
Table 5.1, shows false positive rate, true positive rate, segmentation accuracy, specificity and area under curve results of all 20 test images of DRIVE databases using threshold based on Equal Error Rate (EER).

Table 5.1: Performance of the Proposed Segmentation Method for 20 Images of DRIVE Database, Using Threshold Based on EER

\begin{tabular}{|c|c|c|c|c|c|}
\hline Approach 1 & FPR (\%) & TPR (\%) & Accuracy (\%) & SP (\%) & AUC (\%) \\
\hline Image 1 & 9.09 & 91.33 & 90.97 & 90.91 & 96.60 \\
\hline Image 2 & 9.77 & 89.55 & 90.12 & 90.23 & 96.09 \\
\hline Image 3 & 12.37 & 87.33 & 87.58 & 87.63 & 94.34 \\
\hline Image 4 & 10.35 & 88.87 & 89.57 & 89.65 & 95.44 \\
\hline Image 5 & 12.45 & 87.30 & 87.52 & 87.55 & 93.81 \\
\hline Image 6 & 13.19 & 87.33 & 86.89 & 86.81 & 93.59 \\
\hline Image 7 & 12.76 & 87.07 & 87.21 & 87.24 & 93.98 \\
\hline Image 8 & 15.07 & 86.38 & 86.81 & 84.93 & 92.70 \\
\hline Image 9 & 11.24 & 88.24 & 88.69 & 88.76 & 94.52 \\
\hline Image 10 & 11.20 & 88.55 & 88.87 & 88.80 & 94.81 \\
\hline Image 11 & 14.25 & 87.55 & 85.72 & 85.71 & 92.99 \\
\hline Image 12 & 11.07 & 88.18 & 88.83 & 88.93 & 94.80 \\
\hline Image 13 & 11.23 & 87.46 & 88.58 & 88.77 & 94.58 \\
\hline Image 14 & 10.90 & 88.54 & 89.03 & 89.10 & 95.34 \\
\hline Image 15 & 10.35 & 88.87 & 89.57 & 89.65 & 95.44 \\
\hline Image 16 & 9.73 & 89.76 & 90.20 & 90.27 & 95.83 \\
\hline Image 17 & 12.84 & 88.63 & 87.35 & 87.16 & 94.53 \\
\hline Image 18 & 9.15 & 89.85 & 90.85 & 90.73 & 95.86 \\
\hline Image 19 & 7.15 & 92.21 & 92.77 & 92.85 & 97.31 \\
\hline Image 20 & 8.93 & 90.91 & 91.05 & 91.07 & 96.43 \\
\hline Average & $\mathbf{1 1 . 1 5}$ & $\mathbf{8 8 . 7 0}$ & $\mathbf{8 8 . 9 1}$ & $\mathbf{8 8 . 8 4}$ & $\mathbf{9 4 . 9 5}$ \\
\hline
\end{tabular}


Table 5.2, shows false positive rate, true positive rate, segmentation accuracy, specificity and area under curve results of all 20 test images of DRIVE databases using threshold based on Total Error Rate (TER).

Table 5.2: Performance of the Proposed Segmentation Method for 20 Images of DRIVE Database, Using Threshold Based on TER

\begin{tabular}{|c|c|c|c|c|c|}
\hline Approach 2 & FPR (\%) & TPR (\%) & Accuracy (\%) & SP (\%) & AUC (\%) \\
\hline Image 1 & 9.64 & 91.46 & 90.54 & 90.36 & 96.58 \\
\hline Image 2 & 9.17 & 89.21 & 90.57 & 90.83 & 96.21 \\
\hline Image 3 & 10.45 & 85.93 & 88.91 & 89.46 & 94.47 \\
\hline Image 4 & 9.38 & 86.69 & 90.06 & 90.62 & 94.68 \\
\hline Image 5 & 8.10 & 84.14 & 90.76 & 91.90 & 93.91 \\
\hline Image 6 & 9.71 & 84.82 & 89.46 & 90.29 & 93.95 \\
\hline Image 7 & 11.12 & 86.68 & 88.49 & 88.78 & 94.28 \\
\hline Image 8 & 14.83 & 86.02 & 85.28 & 85.17 & 92.63 \\
\hline Image 9 & 10.40 & 88.13 & 89.41 & 89.60 & 94.83 \\
\hline Image 10 & 10.14 & 87.59 & 89.57 & 89.86 & 94.91 \\
\hline Image 11 & 11.78 & 84.34 & 87.70 & 88.22 & 93.48 \\
\hline Image 12 & 10.50 & 88.52 & 89.37 & 89.50 & 95.24 \\
\hline Image 13 & 10.36 & 86.91 & 89.23 & 89.64 & 94.83 \\
\hline Image 14 & 10.34 & 87.65 & 89.41 & 89.66 & 95.33 \\
\hline Image 15 & 9.50 & 88.99 & 90.33 & 90.50 & 95.80 \\
\hline Image 16 & 8.99 & 89.18 & 90.76 & 91.01 & 96.02 \\
\hline Image 17 & 10.64 & 86.61 & 88.99 & 89.36 & 94.58 \\
\hline Image 18 & 86.60 & 89.91 & 91.16 & 91.34 & 96.28 \\
\hline Image 19 & 66.50 & 92.07 & 93.18 & 93.35 & 97.44 \\
\hline Image 20 & 10.26 & 92.63 & 90.08 & 89.74 & 96.60 \\
\hline Average & $\mathbf{1 6 . 4 1}$ & $\mathbf{8 7 . 7 7}$ & $\mathbf{8 9 . 6 6}$ & $\mathbf{8 9 . 9 5}$ & $\mathbf{9 5 . 1 0}$ \\
\hline
\end{tabular}


Table 5.3, shows false positive rate, true positive rate, segmentation accuracy, specificity and area under curve results of all 20 test images of DRIVE databases using threshold based on maximum accuracy.

Table 5.3: Performance of the Proposed Segmentation Method for 20 Images of DRIVE Database, Using Threshold Based on Maximum Accuracy

\begin{tabular}{|c|c|c|c|c|c|}
\hline Approach 3 & FPR $(\%)$ & TPR $(\%)$ & Accuracy $(\%)$ & SP $(\%)$ & AUC $(\%)$ \\
\hline Image 1 & 2.69 & 72.94 & 93.93 & 97.31 & 96.58 \\
\hline Image 2 & 2.47 & 73.36 & 93.72 & 97.53 & 96.21 \\
\hline Image 3 & 2.78 & 68.60 & 92.71 & 97.22 & 94.48 \\
\hline Image 4 & 2.03 & 67.42 & 93.60 & 97.97 & 94.68 \\
\hline Image 5 & 2.49 & 70.05 & 93.47 & 97.51 & 93.91 \\
\hline Image 6 & 1.97 & 65.49 & 93.11 & 98.03 & 93.95 \\
\hline Image 7 & 1.89 & 63.85 & 93.32 & 97.03 & 94.28 \\
\hline Image 8 & 2.97 & 57.40 & 91.75 & 97.34 & 92.81 \\
\hline Image 9 & 2.66 & 68.84 & 93.71 & 97.74 & 94.83 \\
\hline Image 10 & 2.26 & 68.77 & 93.98 & 98.04 & 94.91 \\
\hline Image 11 & 1.96 & 62.65 & 93.38 & 97.49 & 93.49 \\
\hline Image 12 & 2.51 & 68.24 & 93.56 & 97.23 & 95.24 \\
\hline Image 13 & 2.77 & 69.51 & 93.07 & 97.52 & 94.82 \\
\hline Image 14 & 2.48 & 67.21 & 93.72 & 97.53 & 95.53 \\
\hline Image 15 & 1.92 & 67.60 & 94.72 & 98.08 & 95.80 \\
\hline Image 16 & 2.58 & 73.09 & 94.08 & 97.42 & 96.02 \\
\hline Image 17 & 2.54 & 66.46 & 93.28 & 97.46 & 94.59 \\
\hline Image 18 & 2.29 & 69.84 & 94.31 & 97.71 & 96.28 \\
\hline Image 19 & 2.23 & 81.25 & 95.60 & 97.77 & 97.44 \\
\hline Image 20 & 2.69 & 70.42 & 94.20 & 97.31 & 96.60 \\
\hline Average & $\mathbf{2 . 4 1}$ & $\mathbf{6 8 . 6 5}$ & $\mathbf{9 3 . 6 6}$ & $\mathbf{9 7 . 5 6}$ & $\mathbf{9 5 . 1 2}$ \\
\hline
\end{tabular}


According the averages of parameters in three proposed approaches, we can realize that the proposed segmentation method using threshold based on maximum accuracy with decreasing of FPR, and increasing of accuracy, specificity, and maximizing of the area under curve has the best performance among all three approaches.

Table 5.4 shows the average accuracy, specificity and area under curve of proposed three approaches on twenty images from DRIVE database. The results indicate that threshold determination in approach 3 generates the best performance in terms of segmentation performance measures of Accuracy, Specificity and AUC.

Table 5.4: Performance Accuracy, Specificity and Area Under Curve of the Retina Blood Vessels Achieved by Three Different Approaches

\begin{tabular}{|ll|c|c|c|}
\hline \multicolumn{2}{|c|}{ Threshold Selection } & Accuracy (\%) & Specificity (\%) & AUC (\%) \\
\hline Approach 1 (EER) & 88.91 & 88.83 & 94.95 \\
\hline Approach 2 (TER) & 89.66 & 89.95 & 95.1 \\
\hline Approach 3 (max accuracy) & $\mathbf{9 3 . 6 6}$ & $\mathbf{9 7 . 5 6}$ & $\mathbf{9 5 . 1 2}$ \\
\hline
\end{tabular}

It can be seen that the proposed approach 3 performs better with higher accuracy, specificity and area under curve than other proposed approaches.

We compare the performance of the proposed Gabor filtering method, with the alternative methods available in the literature. Table 5.5 shows the Accuracy performance our segmentation along with those reported for previous segmentation algorithms tested. The results are calculated by the average 20 test images from DRIVE databases for evaluation. 
Table 5.5: Vessels Segmentation Accuracy of the Proposed Method and the Alternative Methods in the Literature

\begin{tabular}{|l|c|}
\hline Method & Accuracy (\%) \\
\hline Ricci [33] & 95.63 \\
\hline Mendonca [34] & 94.63 \\
\hline IUWT [41] & 93.71 \\
\hline Proposed Method with Approach 3 & $\mathbf{9 3 . 6 6}$ \\
\hline Grag [36] & 93.61 \\
\hline Espona [37] & 93.52 \\
\hline Martinez-Perez [38] & 93.44 \\
\hline
\end{tabular}

The results in Table 5.5 show that proposed Gabor based detection and segmentation method using threshold determination by using approach 3 is better than some of the methods in the Table. It can be seen proposed segmentation method using threshold based on maximum accuracy achieved an accuracy score of 93.66 and is higher than the results of Grag [36], Espona [37] and Martinez-Perez [38] vessel segmentation methods.

Table 5.6 indicates the area under curve of blood vessels detection in the retina obtained by different recently reported methods, in terms of AUC values, using the same set of 20 images in the test set of the DRIVE database. 
Table 5.6: The Area Under Curve of the Proposed Method and the Alternative Methods in the Literature

\begin{tabular}{|l|c|}
\hline Dtection Method & AUC (\%) \\
\hline Multiscale Gabor filters; Soares et al. [39] & 96 \\
\hline Multiscale Gabor filters; [29] & 96 \\
\hline proposed Method With Approach 3 & $\mathbf{9 5}$ \\
\hline Single-scale Gabor filters; Rangayyan et al. [31] & 95 \\
\hline Ridge-based segmentation; Staal et al. [1] & 95 \\
\hline Adaptive local thresholding; Jiang and Mojon [40] & 93 \\
\hline Matched filter; Chaudhuri et al. [41] & 91 \\
\hline
\end{tabular}

The results obtained by Table 5.5 indicates the Area Under Curve of the proposed segmentation method using threshold based on maximum accuracy by score of 95 is higher than the results of the Adaptive local thresholding; Jiang and Mojon [40] by score 93 and Matched filter; Chaudhuri et al. [41] by score 91 .

We have compared three approaches for false positive rate, true positive rate, segmentation accuracy and specificity in Figures 5.1 to 5.4 for image 01 of DRIVE database.

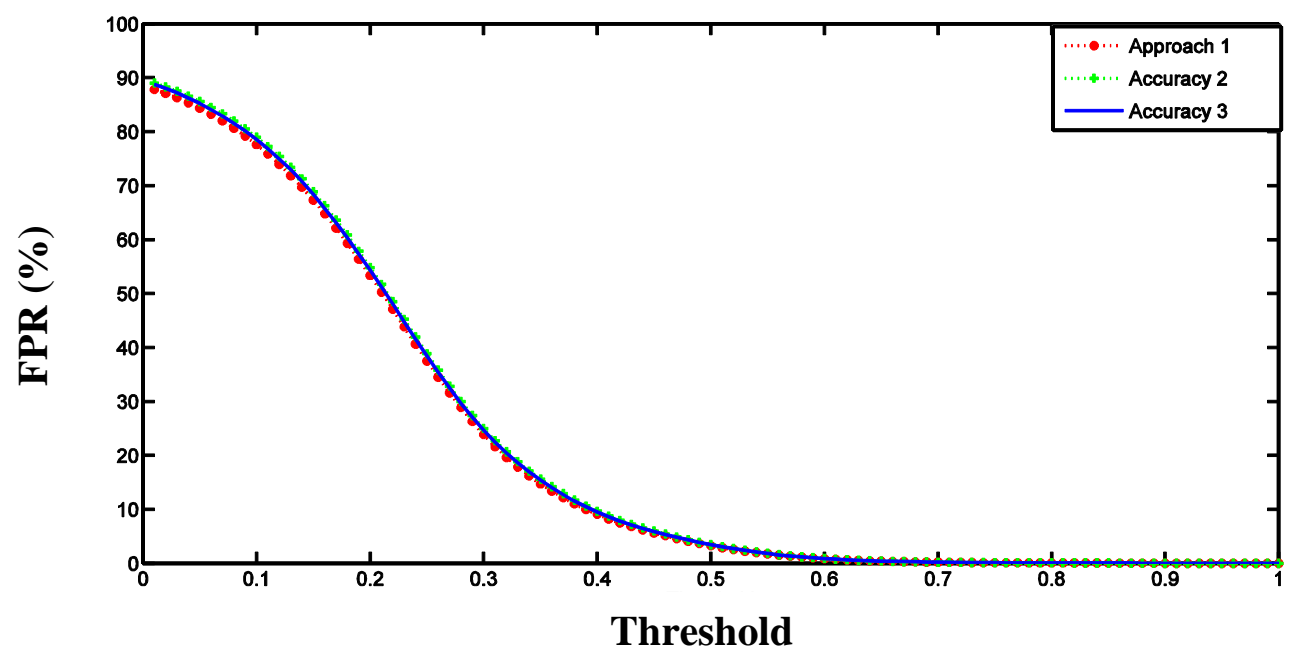

Figure 5.1: The False Positive Ratio Corresponding to Threshold for Three Different Approaches 
Figure 5.1 indicates the False Positive Ratio (FAR) corresponding to threshold for 3 different approaches. The results show that performance of the proposed segmentation method using threshold based on maximum accuracy, by FPR $=2.41 \%$ has minimum FPR in performance.

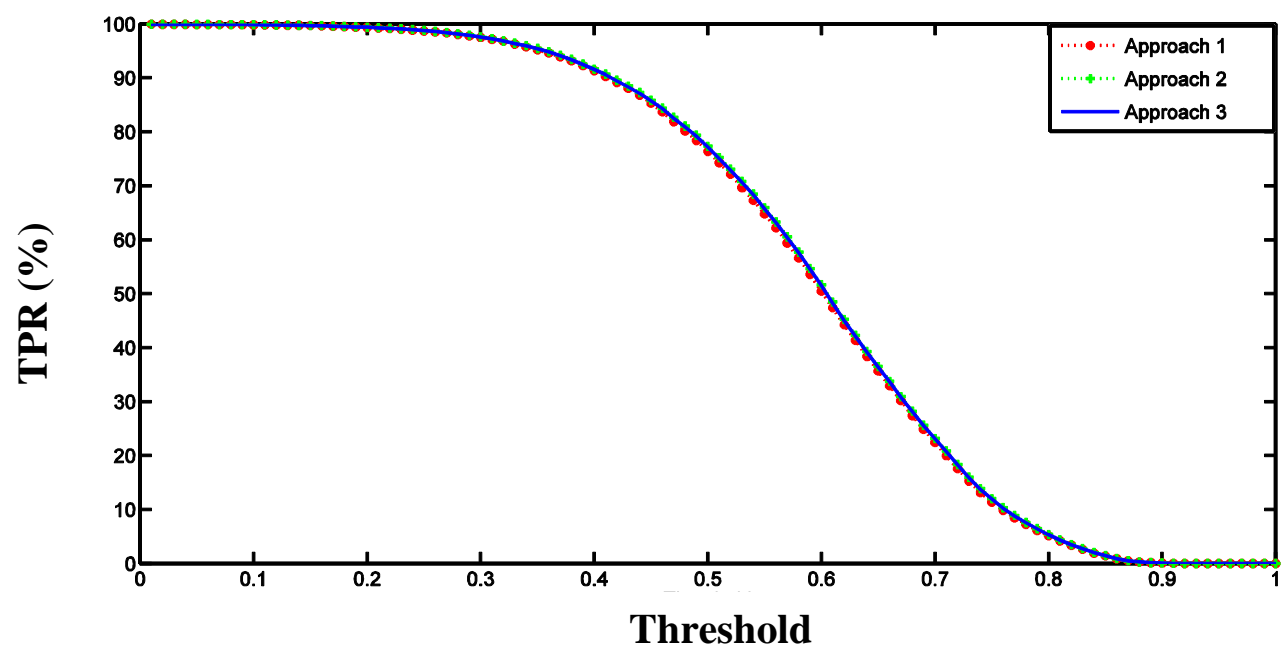

Figure 5.2: The True Positive Ratio Corresponding to Threshold for Three Different Approaches

Figure 5.2 indicates the True Positive Ratio (TPR) corresponding to threshold for 3 different approaches. The results show that performance of the proposed segmentation method using threshold based on Equal Error Rate (EER ), by $\mathrm{TPR}=88.96 \%$ has maximum sensitivity in performance. 


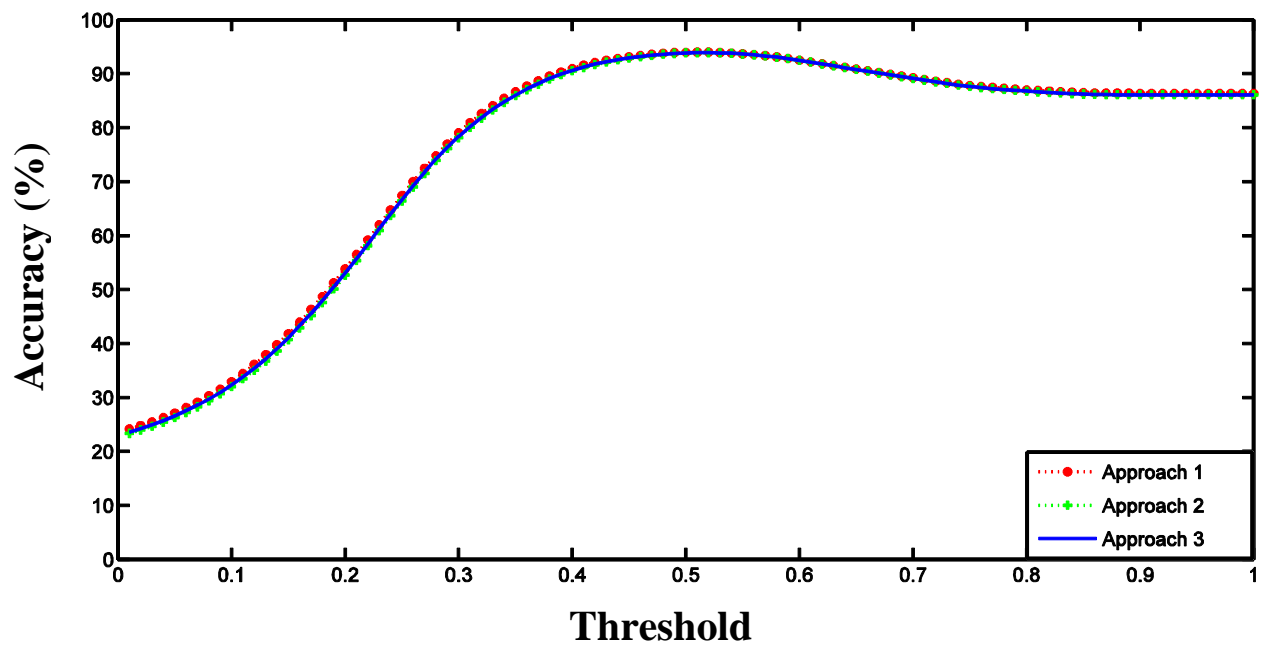

Figure 5.3: The Accuracy Corresponding to Threshold for Three Different Approaches

Figure 5.3 indicates the accuracy corresponding to threshold for 3 different approaches. The results show that performance of the proposed segmentation method using threshold based on maximum accuracy, has maximum accuracy $93.66 \%$ in performance.

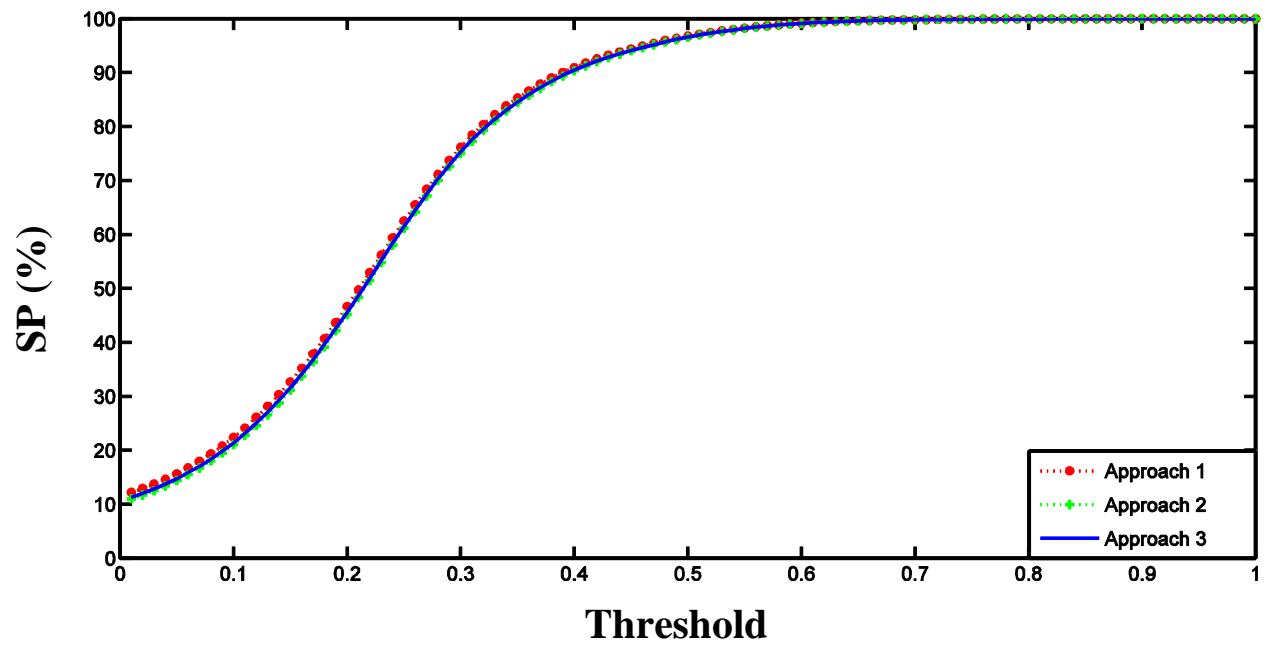

Figure5.4: The Specificity Corresponding to Threshold for Three Different Approaches 
Figure 5.4 indicates the specificity (SP) corresponding to threshold for 3 different approaches. The results show that performance of the proposed segmentation method using threshold based on maximum accuracy, by $\mathrm{SP}=97.65 \%$ has maximum precision in performance.

\subsection{Analysis of Changing Number of Directions in Gabor Filters}

In blood vessels detection using Gabor filters, we have utilized 180 different directions. In this part number of directions has been changed and performances of three parameters have been considered. Table 5.7, represents true positive rate (TPR), segmentation accuracy and area under curve (AUC) results corresponding to changing number of directions among all 180 directions for image19 of test DRIVE databases by proposed approach 3 . 
Table 5.7: Result of Changing Number of Direction in Gabor Filter for Image 19 of DRIVE Database According Approach 3

\begin{tabular}{|c|c|c|c|}
\hline $\begin{array}{l}\text { Number Of } \\
\text { Direction }\end{array}$ & TPR (\%) & ACCURACY (\%) & $\operatorname{AUC}(\%)$ \\
\hline 2 & 52.42 & 92.06 & 87.13 \\
\hline 4 & 74.83 & 94.9 & 96.11 \\
\hline 8 & 78.64 & 95.69 & 97.33 \\
\hline 12 & 79.28 & 95.63 & 97.43 \\
\hline 16 & 80.17 & 96.64 & 97.44 \\
\hline 20 & 80.78 & 95.63 & 97.44 \\
\hline 28 & 81.16 & 95.6 & 97.44 \\
\hline 32 & 81.15 & 95.6 & 97.44 \\
\hline 38 & 81.19 & 95.6 & 97.44 \\
\hline 40 & 81.25 & 95.6 & 97.44 \\
\hline 48 & 79.25 & 95.6 & 97.44 \\
\hline 54 & 79.29 & 95.6 & 97.44 \\
\hline 58 & 79.31 & 95.59 & 97.44 \\
\hline 64 & 79.31 & 95.59 & 97.44 \\
\hline 120 & 79.37 & 95.59 & 97.44 \\
\hline 140 & 79.37 & 95.59 & 97.44 \\
\hline 160 & 79.37 & 95.59 & 97.44 \\
\hline 180 & 79.37 & 95.59 & 97.44 \\
\hline
\end{tabular}

Presentation of True Positive Ratio (TPR), accuracy and Area Under Curve (AUC) against to changing number of direction from $1^{\circ}$ to $180^{\circ}$ degree in Gabor filter, based on results in Table 5.7 have been indicated in Figures 5-5 to 5-7, respectivy. 


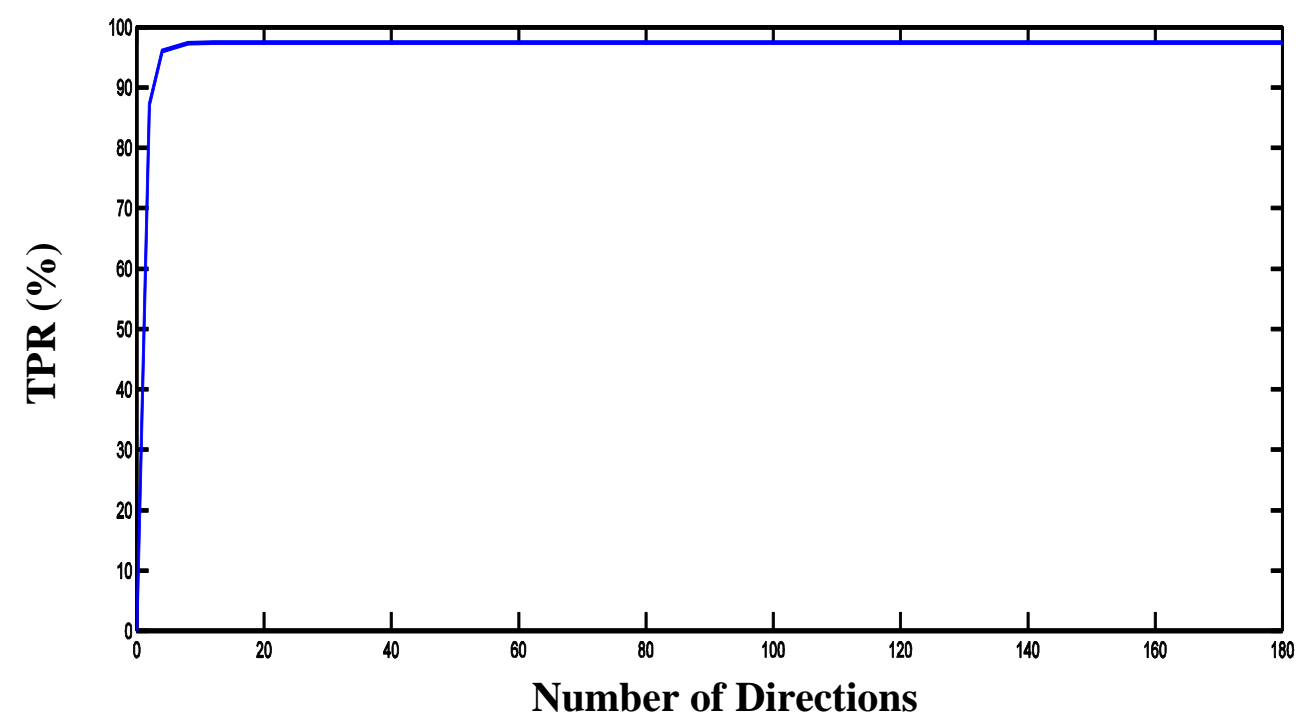

Figure 5.5: The True Positive Ratio Corresponding to Number of Direction in Image 19 of DRIVE Database

Figure 5.5 shows the True Positive Ratio (TPR) against to changing number of directions in image 19 from DRIVE database. It can be seen TPR value beyond number of direction 48 did not change.

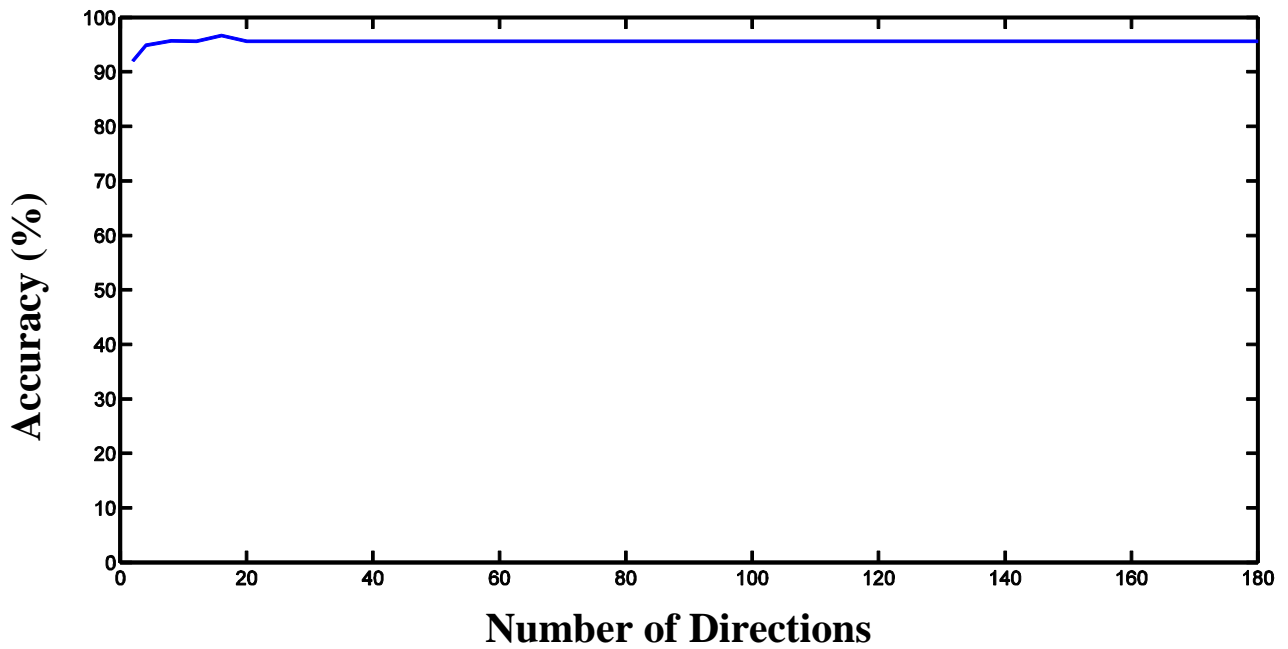

Figure 5.6: The Accuracy Corresponding to Number of Direction in Image 19 of DRIVE Database 
Figure 5.6 shows the accuracy against to changing number of directions in image 19 from DRIVE database. It is found that increasing the number of direction of Gabor filters beyond 28 did not change result in high increase of vessel detection accuracy.

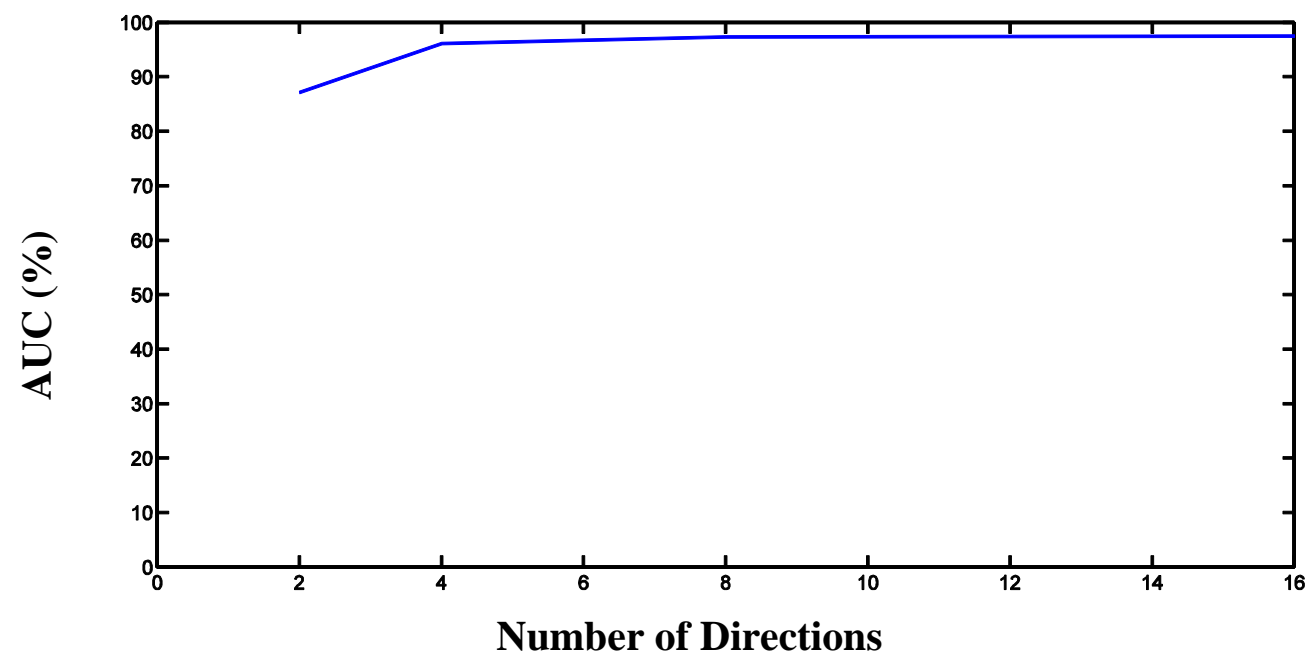

Figure 5.7: The Area Under Curve Corresponding to Number of Direction in Image 19 of DRIVE Database

Figure 5.7 shows the Area Under Curve (AUC) against to changing number of directions in image 19 from DRIVE database. It can be seen AUC value beyond number of direction 16 did not change.

\subsection{Thickness and Elongation Values in Gabor filter}

In previous chapter we have explained the Gabor filter parameters. $\tau$ represents the thickness of the line detector and $\mathrm{l}$ determins the elongation of Gabor filter. Experiments are concluded with $\tau=\{1,2,3,4,5,6,7, \ldots, 14,15,16\}$ and $\mathrm{l}=$ 
$\{1.3,1.7,2.1,2.5,2.9,3.3,3.7,4.1\}$. In this part we have considered area under curve result corresponding to variety of thickness and elongation, respective.

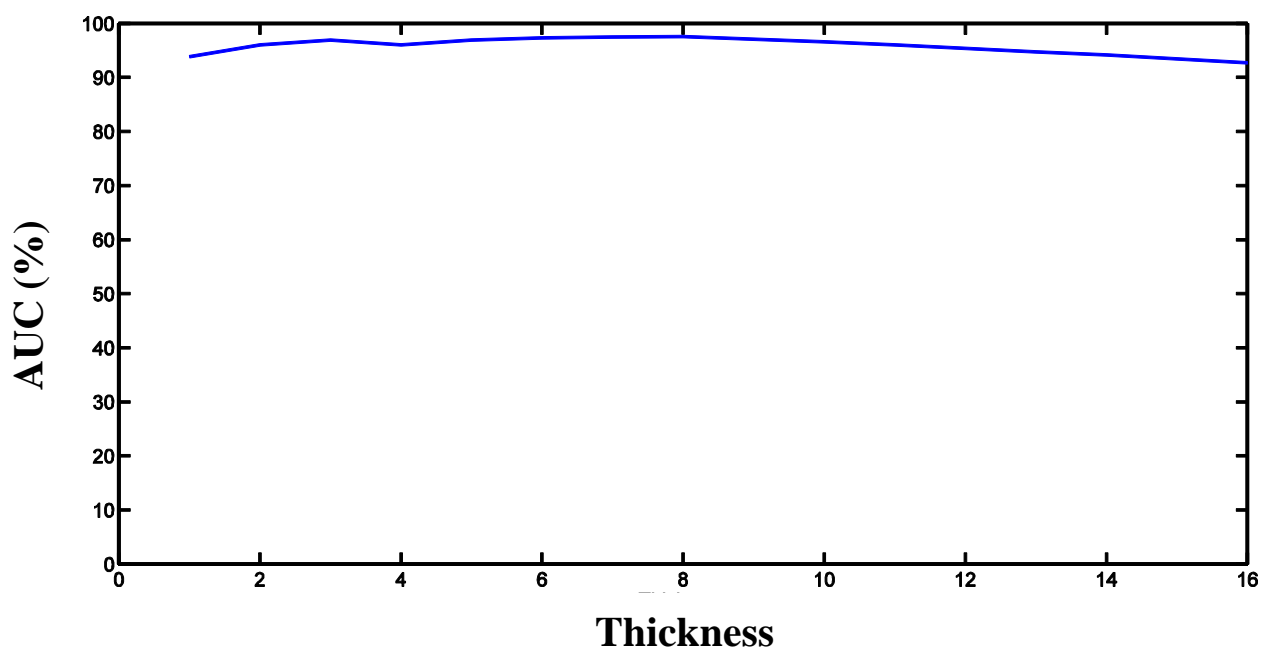

Figure 5.8: The Area Under Curve Corresponding to Thickness of Gabor Filter in Image 19 of DRIVE Database

Figure 5.8 indicates Area Under Curve (AUC) corresponding to thickness of Gabor filter for image 19 test of DRIVE databases. Thickness of Gabor filter in range of 1 to 16 has been changed. Based on formation, we can realize that maximum result of Area Under Curve (AUC) happens, in point by thickness 8.

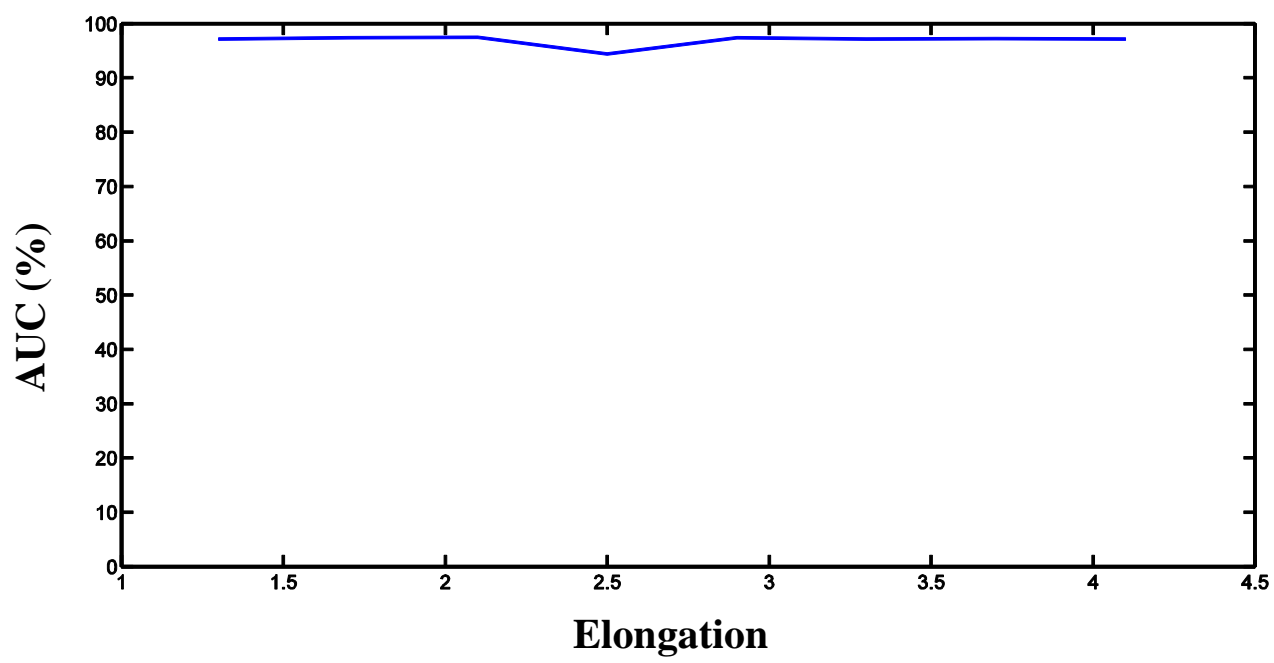


Figure 5.9: The Area Under Curve Corresponding to Elongation of Gabor Filter in Image 19 of DRIVE Database

Figure 5.9 indicates Area Under Curve (AUC) corresponding to elongation of Gabor filter for image 19 test of DRIVE databases. Elongation of Gabor filter has been changed in range of 1.3 to 4.1 . Based on formation, we can realize that maximum results of Area Under Curve (AUC) in Gabor filter is with elongation values 2.1 and 2.7.

Table 5.8 indicates relation between thickness and elongation in Area Under Curve (AUC).The result is shown that Gabor filter with $\tau=7$ and $l=2.5$ has maximum of AUC.

Table 5.8: Relation between Thickness and Elongation in Area Under Curve (AUC)

\begin{tabular}{|c|c|c|c|c|c|c|}
\hline AUC & $l=1.7$ & $l=2.1$ & $l=2.5$ & $l=2.9$ & $l=3.3$ & $l=3.7$ \\
\hline$\tau=1$ & 95.53 & 95.55 & 95.52 & 95.49 & 95.46 & 95.41 \\
\hline$\tau=2$ & 88.38 & 89.02 & 89.73 & 90.11 & 90.38 & 90.58 \\
\hline$\tau=3$ & 92.97 & 93.63 & 94.37 & 94.76 & 95.04 & 95.27 \\
\hline$\tau=4$ & 95.52 & 95.91 & 96.32 & 96.53 & 96.67 & 96.77 \\
\hline$\tau=5$ & 96.58 & 96.82 & 97.06 & 97.16 & 97.20 & 97.23 \\
\hline$\tau=6$ & 97.13 & 97.27 & 97.36 & 97.40 & 97.37 & 97.31 \\
\hline$\tau=7$ & 97.37 & 97.43 & 97.44 & 97.38 & 97.29 & 97.18 \\
\hline$\tau=8$ & 97.38 & 97.38 & 97.28 & 97.15 & 96.97 & 96.77 \\
\hline$\tau=9$ & 97.15 & 97.09 & 96.88 & 96.67 & 96.43 & 96.19 \\
\hline$\tau=10$ & 96.78 & 96.64 & 96.34 & 96.07 & 95.78 & 95.49 \\
\hline$\tau=11$ & 96.27 & 96.07 & 95.70 & 95.39 & 95.07 & 94.74 \\
\hline$\tau=12$ & 95.71 & 95.48 & 95.06 & 94.70 & 94.32 & 93.96 \\
\hline$\tau=13$ & 95.12 & 94.86 & 94.39 & 93.97 & 93.56 & 93.19 \\
\hline$\tau=14$ & 94.53 & 94.23 & 93.69 & 93.24 & 92.81 & 92.45 \\
\hline$\tau=15$ & 93.87 & 93.56 & 92.97 & 92.48 & 92.06 & 91.71 \\
\hline$\tau=16$ & 93.17 & 92.83 & 92.16 & 91.67 & 91.25 & 90.92 \\
\hline
\end{tabular}




\section{Chapter 6}

\section{CONCLUSIONS AND FUTURE WORK}

\subsection{Conclusions}

In this work we proposed to use Gabor filters for the detection of blood vessels in retina images. 180 filters with 1 degree of separation in their orientation are used to capture the details of the vessels for further processing. The pixel values on the detected images are determined by choosing the maximum response corresponding to each pixel from the 180 different filters. Once the detection is over, segmentation process which utilizes a threshold level for segmentation is employed. One of the most important issues is the method in which the threshold value is determined. We have employed three approaches to determine an effective threshold for the segmentation process. Among these the threshold determination approaches, the approach which maximizes the accuracy of the segmentation is found to be generating the highest performing threshold value.

In addition, the thesis studies the effect of changing parameters of the Gabor filter on the final segmentation performance. The parameters such as the elongation and thickness of the Gabor filter are studied for fine tuning of the filters. Furthermore, the number of directions to be used in the filtering process is also studied. 
The generated results are compared with the results available in the literature and the findings show that the results of the proposed approach are comparable with the results reported in the literature. The threshold value selection in most references is not systematic. Typically, heuristic approaches such as experimental analysis are used to determine a threshold value. It is often not mentioned, what these experimental analysis methods are. In this thesis, we introduced a systematic way of determining the threshold value. Namely, EER, TER and Accuracy minimization approaches are introduced to determine the threshold.

\subsection{Future Work}

Current study is determining the threshold values in a supervised manner. That is to say the threshold values are optimized by using manually segmented samples. An unsupervised method can be developed such that threshold can be determined automatically which would be independent of the ground truth. Methods such as entropy of the input retina image can be suiTable to determine a reliable threshold in an unsupervised manner. An extensive study is required on a larger retina database for improved performance. 


\section{REFERENCES}

[1] J. Staa, M.D. Abràmoff, M. Niemeijer, MA. Viergever, and B. van Ginneken, "Ridge-based vessel segmentation in color images of the retina," IEEE Transactions on Medical Imaging, vol. 23(4), pp. 501-509, 2004.

[2] A. Hoover and M. Goldbaum, "Locating the optic nerve in a retinal image using the fuzzy," IEEE Transactions on Medical Imaging, vol. 22(8), pp. 951-958, 2003.

[3] J.V.C. Soares, J.J.G. Leandro, J.R. Cesar, H.F.Jelinek, and M.J Cree, "Retinal vessel segmentation using the 2-D Gabor wavelet and supervised classification," IEEE Transactions on Medical Imaging, vol. 25, pp. 1214-1222, 2006.

[4] B. Kochner, D. Schuhmann, M. Michaelis, G. Mann , and KH Englmeier., "Course tracking and contour extraction of retinal vessels from color fundus photographs: most efficient use of steerable filters for model based image analysis, "Proceedings of the SPIE, The International Society for Optical Engineering,, San Diego, CA, 1998.

[5] C. nthanayothin, JF. Boyce,HL. Cook, and TH. Williamson, "Automated localisation of the optic disc, fovea, and retinal blood vessels from digital colour fundus images, " British Journal of Ophthalmology, vol. 83(4), pp. 902-910, 1999. 
[6] PH. Gregson, Z. Shen, RC. Scott, and V. Kozousek, "Automated grading of venous beading, " Computers and Biomedical Research, August 1995.

[7] F. Oloumi and R. Rangayyan, "Detection of the temporal arcade in fundus images of the retina using the Hough transform, " Engineering in Medicine and Biology Society,Annual International Conference of the IEEE, September 2009.

[8] R. Gelman, ME. Martinez-Perez, DK. Vanderveen , A. Moskowitz, and AB Fulton, "Diagnosis of Plus disease in retinopathy of prematurity using retinal image multiscale analysis," Investigative Ophthalmology \& Visual Science, vol. 46, no. 12, p. 4734-4738, 2005.

[9] "DRIVE: Digital Retinal Images for Vessel Extraction," [Online]. Available: http:// www. isi.uu.nl/ Research/Databases/ DRIVE/.

[10] P. C. Siddalingaswamy, K. Gopalakrishna Prabhu, "Automatic detection of multiple oriented blood vessels in retinal images," J. Biomedical Science and Engineering, vol. 3, pp. 101-107, 2010.

[11] V. B. Soares and M. Cesar Jr Roberto, "Retinal Vasculature Segmentation UsingWavelets and Supervised Classification," annias de congresso da SBC, 2007. 
[12] Stiles, G. Wyszecki and W. S, "Color science: Concepts and methods, quantitative data and formulae," New York, 1982.

[13] J. Jonas, U. Schneider, and G. Naumann, "Count and density of human retinal photoreceptors," in pages 230:505-510, 1992.

[14] X. Zhu, R.M. Rangayyan, and A.L. Ells, "Digital Image Processing for Ophthalmology," 2011.

[15] Q. Li, J. You, L. Zhang, and D. Zhang, "A New Approach to Automated Retinal Vessel Segmentation Using Multiscale Analysis," in The 18th International Conference on Pattern Recognition (ICPR'06), 2006.

[16] D. Gabor, "Theory of communication," Journal of the Institute of Electrical Engineers, pp. 429-457, 1946.

[17] J. Daugman, "Uncertainty relation for resolution in space, spatial frequency and orientation optimized by two-dimensional visual cortical filters," J. Opt. Soc. Am. A, vol. 2, no. 7, pp. 1160-1 169, July, 1985.

[18] S. Marcelja, "Mathematical description of the response of simple cortical cells," J. Opt.Soc, p. 1297-1300, 1980.

[19] M. A. Bastiaans, "A sampling theorem for the complex spectrogram and 
Gabor's expansion of a signal in Gaussian elementary signals," Optica Acta, vol. 20, no. 4, p. 594-598, 1981.

[20] Zeevi, M. Porat and Y.Y, "The generalized gabor scheme of image representation in biological and machine vision," IEEE Transactions on PAMI, vol. 10, no. 4, p. 452-468, 1988.

[21] T. Lee, "Image representation using 2d gabor-wavelets," IEEE Transactions on PAMI, vol. 18, no. 10, p. 959-971, 1996.

[22] D.K. Solanki and K. Bhoi, "Texture Segmentation Using Optimal Gabor Filter," National Institute of Technology Rourkela, India, 2011.

[23] J. Movellan, "Tutorials on Gabor Filters," pp.1-20,GNU Free documentation License 1.1,Kolmogorv Project, 2002.

[24] M. Misiti, Y. Misiti, G. Oppenheim, and J. Poggi, "Wavelet Toolbox User's Guide," mathworks, 2012.

[25] A. KRISHAN, "Evaluation of Gabor filter parameters for image enhancement and segmentation, " THAPAR UNIVERSITY, 2009.

[26] Woods, RC. Gonzalez and RE, Digital Image Processing, Prentice Hall, 2002. 
[27] J. Canny, "A computational approach to edge detection, " IEEE Transactions on Pattern Analysis and Machine Intelligence, vol. 8, no. 6, p. 670-698, 1986.

[28] L. K. Westin, "Receiver operating characteristic (ROC) analysis," ISSN-03480542, Sweden.

[29] F. Oloumi, R. M. Rangayyan, F. Oloumi, P. Eshghzadeh-Zanjani, and F. J. Ayres, "Detection of Blood Vessels in Fundus Images of the Retina using Gabor Wavelets, "Proceedings of the 29th Annual International Conference of the IEEE EMBS, Lyon, France, 2007.

[30] "Structured Analysis of the Retina," 24 Maech 2008. [Online]. Available: http:// www. ces. clemson. edu / ahoover/ stare/.

[31] RM. Rangayyan, F. Oloumi , F. Oloumi, P. Eshghzadeh-Zanjani, FJ. Ayres, "Detection of blood vessels in the retina using Gabor filter, " Proceedings of the 20th Canadian Conference on Electrical and Computer Engineering (CCECE 2007), Canada, 2007.

[32] F. FJ Ayres and RM Rangayyan, "Performance analysis of oriented feature detectors, "Proceedings of SIBGRAPI 2005: XVIII Brazilian Symposium on Computer Graphics and Image Processing, Brazil, 2005.

[33] E. Ricci, R. Perfetti, "Retinal blood vessel segmentation using line operators and 
support vector classification," IEEE Trans Med Imaging, vol. 26, p. 1357-1365, 2007.

[34] AM. Mendonc, A. Campilho, "Segmentation of retinal blood vessels by combining the detection of centerlines and morphological reconstruction," IEEE Trans Med Imaging, vol. 25, pp. 1200-1213, 2006.

[35] P. Bankhead, C. Norman Scholfield, J. Graham McGeown, and T. M. Curtis, "Fast Retinal Vessel Detection and Measurement Using Wavelets and Edge Location Refinement," PLoS ONE, vol. 7, no. 3, 2012.

[36] S.Garg, J. Sivaswamy and S. Chandra, "Unsupervised curvature-based retinal vessel segmentation, "4th IEEE International Symposium on Biomedical Imaging: From Nano to Macro, 2007.

[37] L. Espona, MJ. Carreira, MG. Penedo, M. Ortega, "Retinal vessel tree segmentation using a deformable contour mode," 19th International Conference on Pattern Recognition, 2008.

[38] ME. Martinez-Perez, AD. Hughes, SA. Thom, AA. Bharath,KH. Parker,, "Segmentation of blood vessels from red-free and uorescein retinal images," in Med Image Anal 11, 2007.

[39] J. Soares, J. Leandro, J. Cesar . RM, HF. Jelinek, and MJ. Cree, "Retinal vessel 
segmentation using the 2-D Gabor wavelet and supervised classification," IEEE Transactions on Medical Imaging, vol. 25, no. 9, p. 1214-1222, 2006.

[40] X.Jiang and D. Mojon, "Adaptive local thresholding by verification based multithreshold probing with application to vessel detection in retinal images," IEEE Transactions on Pattern Analysis and Machine Intelligence, vol. 25, no. 1, pp. 131-137, 2003.

[41] S. Chaudhuri, S. Chatterjee, N. Katz, M. Nelson, and M. Goldbaum, "Detection of blood vessels in retinal images using two-dimensional matched filters," IEEE Transactions on Medical Imaging, vol. 8, pp. 263-269, 1989. 